\title{
SCEXAO AND GPI $Y J H$ BAND PHOTOMETRY AND INTEGRAL FIELD SPECTROSCOPY OF THE YOUNG BROWN DWARF COMPANION TO HD 1160
}

E. Victor Garcia ${ }^{1,2,3}$, Thayne Currie ${ }^{4}$, Olivier Guyon ${ }^{4,5,6}$, Keivan G. Stassun $^{2,7}$, Nemanja Jovanovic ${ }^{4}$, Julien Lozi 4 Tomoyuki Kudo ${ }^{4}$, Danielle DoughtY ${ }^{8}$, Josh Schlieder ${ }^{9,37}$, J. Kwon ${ }^{10}$, T. Uyama ${ }^{11}$, M. Kuzuhara ${ }^{5,12,13}$, J. C. Carson ${ }^{14}$, T. NaKaGawa ${ }^{10}$, J. Hashimoto ${ }^{5,12}$, N. Kusakabe ${ }^{5,12}$, L. Abe ${ }^{15}$, W. Brandner ${ }^{16}$, T. D. Brandt $^{17,36}$, M. Feldi ${ }^{16}$, M. Goto ${ }^{18}$,

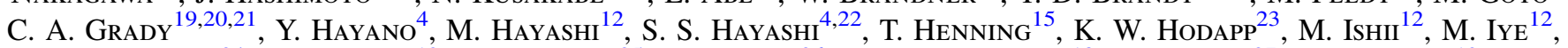

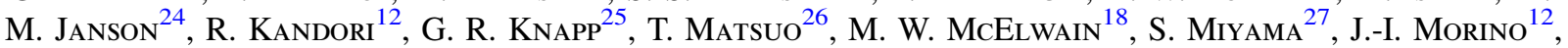
A. Moro-Martin ${ }^{28,29}$, T. Nishimura ${ }^{4}$, T.-S. Pyo ${ }^{4}$, E. Serabyn ${ }^{30}$, T. Suenaga ${ }^{11,22}$, H. Suto $^{5,12}$, R. Suzuki ${ }^{12}$, Y. H. Takahashi ${ }^{12,30}$, H. Takami ${ }^{31}$, M. TaKami ${ }^{31}$, N. Takato ${ }^{4}$ H. Terada $^{12}$, C. Thalmann ${ }^{32}$, E. L. Turner ${ }^{25,33}$, M. Watanabe ${ }^{34}$, J. Wisniewshi ${ }^{35}$, T. Yamada ${ }^{10}$, T. Usuda ${ }^{12}$, AND M. Tamura ${ }^{5,11,12}$

${ }^{1}$ Lowell Observatory, Flagstaff, AZ, 86001, USA; eugenio.v.garcia@gmail.com

${ }^{2}$ Department of Physics and Astronomy, Vanderbilt University, Nashville, TN, USA

${ }^{3}$ Lawrence Livermore National Laboratory, L-210, 7000 East Avenue, Livermore, CA 94550, USA

${ }^{4}$ Subaru Telescope, National Astronomical Observatory of Japan, 650 N. A'ohoku Place, Hilo, HI 96720, USA

${ }^{5}$ Astrobiology Center of NINS, 2-21-1 Osawa, Mitaka, Tokyo, 181-8588, Japan

${ }^{6}$ Steward Observatory, University of Arizona, 933 N Cherry Ave., Tucson, AZ 85721, USA

${ }^{7}$ Department of Physics, Fisk University, Nashville, TN 37208, USA

${ }^{8}$ College of Optical Sciences, University of Arizona, Tucson, AZ 85721, USA

${ }^{9}$ NASA Ames Research Center, Moffett Field, CA, USA

${ }^{10}$ Department of Space Astronomy and Astrophysics, Institute of Space \& Astronautical Science (ISAS), Japan Aerospace Exploration Agency (JAXA), 3-1-1 Yoshinodai, Chuo-ku, Sagamihara, Kanagawa 252-5210, Japan

${ }^{11}$ Department of Astronomy, University of Tokyo, 7-3-1, Hongo, Bunkyo-ku, Tokyo, 113-0033, Japan

${ }_{12}$ National Astronomical Observatory of Japan, 2-21-1, Osawa, Mitaka, Tokyo, 181-8588, Japan

${ }^{13}$ Department of Earth and Planetary Sciences, Tokyo Institute of Technology, Ookayama, Meguro-ku, Tokyo, 152-8551, Japan

${ }^{14}$ Department of Physics and Astronomy, College of Charleston, 58 Coming St., Charleston, SC 29424, USA

${ }^{15}$ Laboratoire Lagrange (UMR 7293), Université de Nice-Sophia Antipolis, CNRS, Observatoire de la Côte d'Azur, 28 Avenue Valrose, F-06108 Nice Cedex 2, France

${ }^{16}$ Max Planck Institute for Astronomy, Königstuhl 17, D-69117 Heidelberg, Germany

${ }^{17}$ Astrophysics Department, Institute for Advanced Study, Princeton, NJ, USA

${ }^{18}$ Universitäts-Sternwarte München, Ludwig-Maximilians-Universität, Scheinerstr. 1, D-81679 München, Germany

${ }^{19}$ Exoplanets and Stellar Astrophysics Laboratory, Code 667, Goddard Space Flight Center, Greenbelt, MD 20771, USA

${ }^{20}$ Eureka Scientific, 2452 Delmer, Suite 100, Oakland, CA 96002, USA ${ }^{21}$ Goddard Center for Astrobiology, USA

22 Department of Astronomical Science, SOKENDAI (The Graduate University for Advanced Studies), 2-21-1 Osawa, Mitaka, Tokyo, 181-8588, Japan

${ }^{23}$ Institute for Astronomy, University of Hawaii, 640 N. A'ohoku Place, Hilo, HI 96720, USA

${ }_{25}^{24}$ Department of Astronomy, Stockholm University, AlbaNova University Center, SE-10691, Stockholm, Sweden

${ }^{25}$ Department of Astrophysical Science, Princeton University, Peyton Hall, Ivy Lane, Princeton, NJ 08544, USA

${ }^{26}$ Department of Earth and Space Science, Graduate School of Science, Osaka University, 1-1 Machikaneyamacho, Toyonaka, Osaka, 560-0043, Japan

${ }_{27}$ Hiroshima University, 1-3-2, Kagamiyama, Higashihiroshima, Hiroshima, 739-8511, Japan

${ }^{28}$ Space Telescope Science Institute, 3700 San Martin Drive, Baltimore, MD 21218, USA

${ }_{30}^{29}$ Center for Astrophysical Sciences, Johns Hopkins University, Baltimore, MD 21218, USA

30 Jet Propulsion Laboratory, California Institute of Technology, Pasadena, CA, 171-113, USA

${ }^{31}$ Institute of Astronomy and Astrophysics, Academia Sinica, P.O. Box 23-141, Taipei 10617, Taiwan

${ }^{32}$ Swiss Federal Institute of Technology (ETH Zurich), Institute for Astronomy, Wolfgang-Pauli-Strasse 27, CH-8093 Zurich, Switzerland

${ }^{33}$ Kavli Institute for Physics and Mathematics of the universe, The University of Tokyo, 5-1-5, Kashiwanoha, Kashiwa, Chiba, 277-8568, Japan

${ }^{34}$ Department of Cosmosciences, Hokkaido University, Kita-ku, Sapporo, Hokkaido, 060-0810, Japan

${ }^{35}$ H. L. Dodge Department of Physics \& Astronomy, University of Oklahoma, 440 W Brooks St Norman, OK 73019, USA

Received 2016 June 24; revised 2016 October 12; accepted 2016 October 16; published 2017 January 11

\section{ABSTRACT}

We present high signal-to-noise ratio, precise $Y J H$ photometry and $Y$ band $(0.957-1.120 \mu \mathrm{m})$ spectroscopy of HD $1160 \mathrm{~B}$, a young substellar companion discovered from the Gemini NICI Planet Finding Campaign using the Subaru Coronagraphic Extreme Adaptive Optics instrument and the Gemini Planet Imager. HD 1160 B has typical mid-M dwarf-like infrared colors and a spectral type of M5.5 $5_{-0.5}^{+1.0}$, where the blue edge of our $Y$ band spectrum rules out earlier spectral types. Atmospheric modeling suggests HD $1160 \mathrm{~B}$ has an effective temperature of $3000-3100 \mathrm{~K}$, a surface gravity of $\log g=4-4.5$, a radius of $1.55 \pm 0.10 R_{\mathrm{J}}$, and a luminosity of $\log L / L_{\odot}=$ $-2.76 \pm 0.05$. Neither the primary's Hertzspring-Russell diagram position nor atmospheric modeling of HD 1160 B show evidence for a subsolar metallicity. Interpretation of the HD 1160 B spectroscopy depends on which stellar system components are used to estimate the age. Considering HD 1160 A, B and C jointly, we derive an age of 80-125 Myr, implying that HD 1160 B straddles the hydrogen-burning limit (70-90 $\left.M_{\mathrm{J}}\right)$. If we consider HD 1160 A alone, younger ages (20-125 Myr) and a brown dwarf-like mass (35-90 $\left.M_{\mathrm{J}}\right)$ are possible. Interferometric measurements of the primary, a precise Gaia parallax, and moderate-resolution spectroscopy can better constrain the system's age and how HD 1160 B fits within the context of (sub)stellar evolution.

\footnotetext{
36 NASA Sagan Fellow.

${ }^{37}$ NASA Postdoctoral Program Fellow.
} 
Key words: instrumentation: adaptive optics - planetary systems - stars: low-mass - techniques: imaging spectroscopy

\section{INTRODUCTION}

In addition to about a dozen super-Jovian-mass extrasolar planets (e.g., Marois et al. 2008; Lagrange et al. 2010; Kraus \& Ireland 2012; Carson et al. 2013; Kuzuhara et al. 2013; Quanz et al. 2013; Rameau et al. 2013; Currie et al. 2014a, 2015; Macintosh et al. 2015), the past decade of ground-based direct imaging observations of young stars has also revealed numerous higher mass (ratio) and (often) wider separation brown dwarf companions (Chauvin et al. 2005; Biller et al. 2010; Ireland et al. 2011). Their typically more modest companion-to-primary contrasts make their detection and precise photometric/spectroscopic calibration possible without having to correct for throughput/signal losses inherent in the algorithms needed to detect more extreme contrast-ratio exoplanets (e.g., Marois et al. 2010). They, and free-floating brown dwarfs of comparable ages, can reveal general trends in infrared colors and spectra that clarify the properties of young substellar atmospheres, can serve as important tests of the same atmosphere models used to characterize bona fide imaged exoplanets, and may provide insights into the formation of substellar objects (e.g., Kratter et al. 2010; Reggiani \& Meyer 2011; Faherty et al. 2016).

One particularly interesting system with a brown dwarf companion is HD 1160, located at a distance of $103.1 \pm 5.3 \mathrm{pc}$ (van Leeuwen 2007) with an A0V primary that is comparable in mass to at least some stars around which planets have been imaged (e.g., $\beta$ Pic). Targeted by the NICI Planet-Finding Campaign (Liu et al. 2010), HD 1160 is orbited by a pair of substellar companions: HD $1160 \mathrm{~B}$ at $\sim 80$ au and HD $1160 \mathrm{C}$ at 530 au (Nielsen et al. 2012, hereafter N12).

N12's photometry for HD $1160 \mathrm{~B}$ and spectrum for HD $1160 \mathrm{C}$ suggested that the former is a $L O \pm 2$ brown dwarf and the latter an M3.5 star. N12 used HD 1160 B's and C's red $J-K_{\mathrm{s}}$ colors, HD 1160 A's luminosity, and the system's large space motion to argue that all components are young: 10-100 Myr. By comparing HD 1160 B and C's derived luminosity to predictions from substellar cooling models given the system's age, N12 derived a mass of 24-45 $M_{\mathrm{J}}$ for HD $1160 \mathrm{~B}$ and $0.18-0.25 M_{\odot}$ for HD $1106 \mathrm{C}$.

HD 1160 B was revisited as part of the first results from the Spectro-Polarimetric High-contrast Exoplanet REsearch (SPHERE, Beuzit et al. 2008) at the Very Large Telescope (VLT) by (Maire et al. 2016, hereafter M16). M16 observed HD 1160 B with VLT/SPHERE to obtain the first spectrum for the object, with resolution of $R \approx 30$ covering the wavelength range of $1.0-1.6 \mu \mathrm{m}$. Based on HD 1160 B's spectral shape, M16 re-classify its spectral type as $M 6_{-0.5}^{+1}$ and derive an effective temperature of $3000 \pm 100 \mathrm{~K}$ but do not constrain the object's surface gravity in their SPHERE $Y J H$ spectrum. Based largely on a wider range and systematically older age estimate (30-300 Myr), they estimate a mass of 39-144 $M_{\mathrm{J}}$ based on bolometric luminosity and 69-172 $M_{\mathrm{J}}$ based on effective temperature.

Two key questions surround the nature of HD 1160's companions. The first is metallicity. By comparing HD 1160 A's Hertzsprung-Russel (HR) diagram position to predictions from the Siess et al. (2000) isochrones, N12 found that the primary is only matched to isochrones with a substantially subsolar metallicity $([\mathrm{Fe} / \mathrm{H}]=-0.3$, in conflict with the very red NIR colors of HD $1160 \mathrm{~B}$ and C. While M16 derived bluer infrared colors for HD $1160 \mathrm{~B}$ and $\mathrm{C}$, they found atmosphere models assuming a subsolar metallicity best fit HD 1160 B's spectrophotometry. As most stars in the solar neighborhood have a near-solar metallicity, new analysis is needed to clarify whether or not HD 1160 A has a non-solar metallicity. The atmospheric properties of HD $1160 \mathrm{~B}$, including metallicity, could be clarified with new spectrophotometry with greater precision, covering a wider wavelength baseline.

The second question is the system's age. Different age estimates for HD 1160 result in quite different inferred masses for the two low-mass companions. HD 1160 B's interpretation changes from a low-mass, high-mass ratio brown dwarf (for a young age) to an object straddling the brown dwarf/stellar boundary (for an older age). The age of the HD 1160 system could be better clarified with a more in-depth and systematic analysis of possible age indicators for the primary and the two companions.

In this work, we present high-precision photometry of the HD 1160 system obtained with the Subaru Coronagraphic Extreme Adaptive Optics (SCExAO) project in the $Y J H$ passbands and integral field spectroscopy with the Gemini Planet Imager (GPI) in the $Y$ band (Section 2). We compare HD $1160 \mathrm{~B}$ 's spectrum and new photometry to that of young brown dwarfs and field objects and match its photometry/spectra using the BT-Settl atmosphere models (Section 3) to address whether or not HD 1160 B clearly shows evidence for a subsolar metallicity, and derive an updated luminosity for both it and HD $1160 \mathrm{C}$. We use an array of age estimates to better clarify the age of the system (Section 4). After modeling HD 1160 B's atmosphere and better constraining HD 1160 C's luminosity, we use our updated age estimate to more precisely infer HD 1160 BC's mass (Section 5). Section 6 summarizes our findings and identifies future work that can better clarify the properties of this system.

\section{DATA}

\subsection{SCEXAO Y JH Imaging}

The SCExAO is a high-contrast imaging platform designed for the discovery and characterization of faint companions (Jovanovic et al. 2015). A partial correction of the low-order modes of the wavefront is performed by the Subaru facility adaptive optics instrument (AO188), and a final correction, including the high-order modes, is performed by a visible-light pyramid wavefront sensor and a 2000-element deformable mirror inside SCExAO. Ultimately, the project is slated to yield $90+\%$ Strehl ratios at $1.6 \mu \mathrm{m}$ on bright stars $(I$ (mag) $<9-10)$.

On 2015 October 31, we observed HD 1160 with SCExAO coupled with the near-infrared (NIR) HiCIAO camera (Tamura 2009) in the $Y, J$, and $H$ broadband filters $(\sim 8.0$ mas pixel $\left.^{-1}\right) .^{38}$ All observations were obtained in angular differential imaging (ADI) mode (Marois et al. 2006) but with a very small amount of field rotation $\left(\Delta\right.$ P.A. $\left.\sim 1{ }^{\circ} .5-2^{\circ}\right)$.

\footnotetext{
38 The plate scale when using AO188 and HiCIAO is 9.5 mas pixel $^{-1}$, but for SCExAO and HiCIAO, the plate scale is 8.3 mas pixel $^{-1}$.
} 

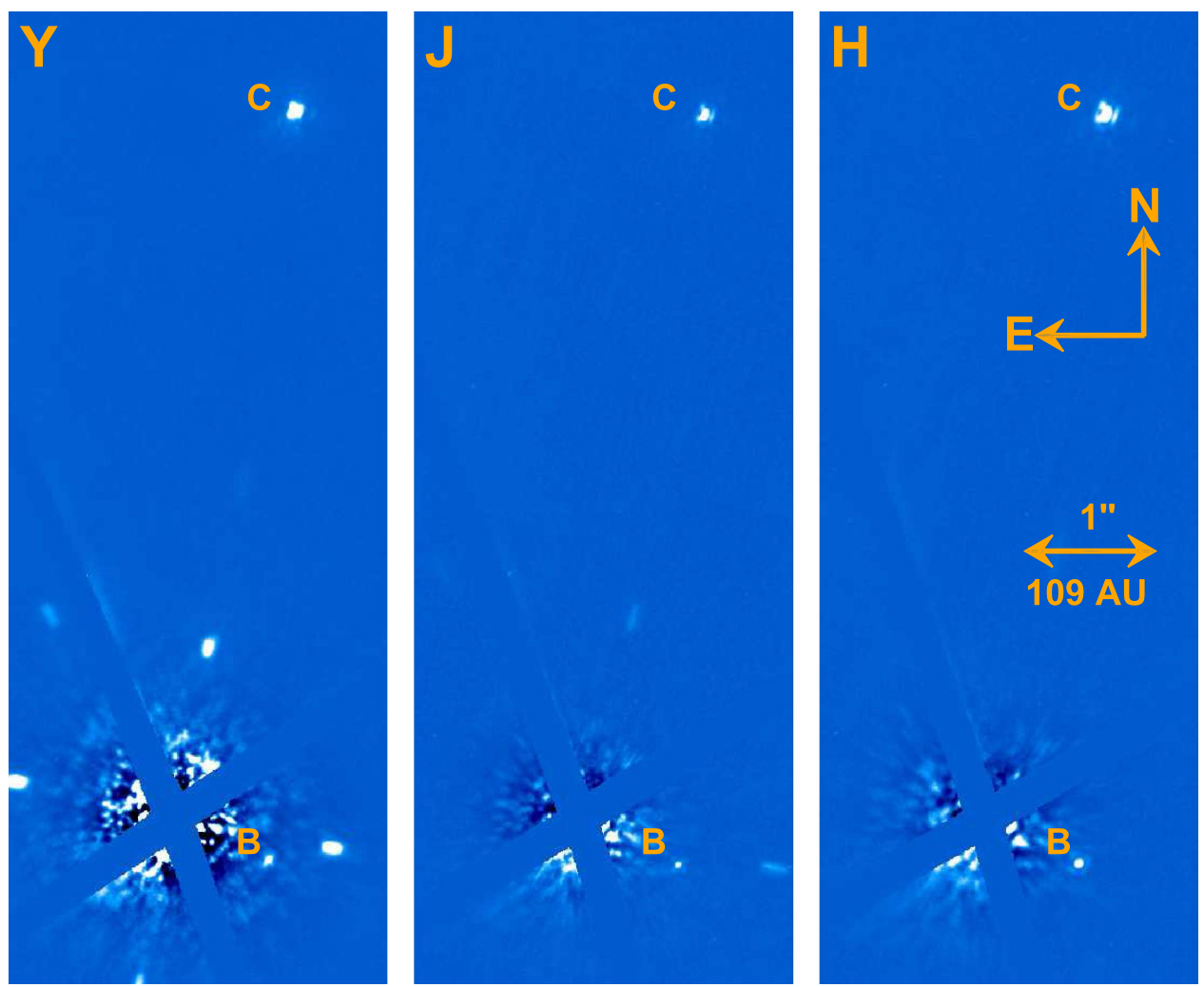

Figure 1. SCExAO observations of HD 1160 in $Y$ (left), $J$ (middle), and $H$ (right). Diffracted light from the secondary spider is masked in each panel. The four bright, elongated speckles $2^{\prime \prime}$ from the star are scattered light from SCExAO, the result of quilting from the deformable mirror, which can be used to aid image registration. The companions HD $1160 \mathrm{~B}$ and $\mathrm{C}$ are detected at a high $\mathrm{S} / \mathrm{N}$ in each image.

Table 1

Observation Log of HD 1160

\begin{tabular}{|c|c|c|c|c|c|c|c|}
\hline Telescope & Instrument & UT Date & Band & $\begin{array}{l}\text { Wavelength } \\
\qquad(\mu \mathrm{m})\end{array}$ & $\begin{array}{l}t_{\text {int }} \\
(\mathrm{s})\end{array}$ & $N_{\exp }$ & Mode \\
\hline Subaru & SCExAO/HiCIAO & 31 Oct 2015 & $Y$ & $0.97-1.07$ & 1.5 & 11 & Photometry \\
\hline Subaru & SCExAO/HiCIAO & $"$ & $Y+\mathrm{ND}$ & $0.97-1.07$ & 1.5 & 6 & Photometry \\
\hline Subaru & SCExAO/HiCIAO & $"$ & $J+\mathrm{ND}$ & $1.17-1.33$ & 1.5 & 5 & Photometry \\
\hline Subaru & SCExAO/HiCIAO & ” & $H$ & $1.49-1.78$ & 1.5 & 5 & Photometry \\
\hline Subaru & SCExAO/HiCIAO & $"$ & $H+\mathrm{ND}$ & $1.49-1.78$ & 1.5 & 5 & Photometry \\
\hline
\end{tabular}

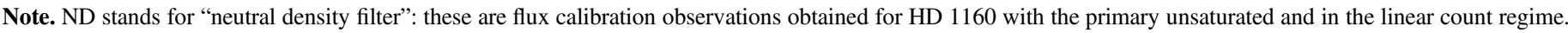
The neutral density filters are $0.388 \pm 0.008 \%, 0.590 \pm 0.004 \%$ and $0.854 \pm 0.002 \%$ for $Y, J$, and $H$, respectively.

While our instantaneous Strehl ratios were in the 80\%-90\% range, strong low-frequency vibrations induced by the telescope drive system degraded AO correction quality to $\sim 60 \%-70 \%$ Strehl over the course of $>0.1 \mathrm{~s}$ observations. These vibrations are now partially mitigated by a linear-quadratic-Gaussian controller, yielding slightly improved performance (Lozi et al. 2016). Upcoming improvements-using the higher-stroke AO188 deformable mirror driven by multiple accelerometers to offload tip-tilt variations-should eliminate these vibrations. Nevertheless, the partial correction offered by SCExAO substantially improves upon the image quality achieved with discovery images of HD 1160 B obtained in the NIR from Gemini/NICI.

Our observing log is detailed in Table 1 . We cycled through the three NIR passbands using a combination of short, lightly saturated exposures and unsaturated images obtained using
HiCIAO's neutral density filters. Basic image processing followed standard methods employed in Currie et al. (2011, 2014a) for flat fielding, dark subtraction, de-striping, radial profile subtraction, and combining images. As HD $1160 \mathrm{~B} / \mathrm{C}$ were plainly visible in the raw data, we did not perform any point-spread function (PSF) subtraction but instead simply removed the azimuthally averaged seeing halo.

Despite the short integration times and no PSF subtraction techniques applied, both HD $1160 \mathrm{~B}$ and $\mathrm{C}$ were easily recovered with a signal-to-noise ratio $(\mathrm{S} / \mathrm{N})$ of $\sim 70$ and $>200$, respectively, in the $Y J H$ passbands (Figure 1). While processing the data with advanced PSF subtraction techniques like A-LOCI or KLIP (Soummer et al. 2012; Currie et al. 2015) yielded stronger detections and lower residuals at smaller separations, they also partially attenuated the companion 
Table 2

Near-to-mid-IR Photometry of HD $1160 \mathrm{~B}$ and C

\begin{tabular}{|c|c|c|c|c|c|c|}
\hline Object & $\begin{array}{l}\text { Band } \\
(\mathrm{MKO})\end{array}$ & $\begin{array}{l}\text { SCExAO/HiCIAO } \\
\text { (mag) }\end{array}$ & $\begin{array}{l}\text { GEMINI/GPI } \\
\text { (mag) }\end{array}$ & $\begin{array}{l}\text { VLT/SPHERE } \\
\text { (mag) }\end{array}$ & $\begin{array}{c}\text { Gemini/NICI } \\
(\mathrm{mag})\end{array}$ & $\begin{array}{c}\text { Keck/NIRC2 } \\
\text { (mag) }\end{array}$ \\
\hline \multirow[t]{5}{*}{ HD $1160 \mathrm{~B}$} & $Y$ & $15.30 \pm 0.04^{\mathrm{a}}$ & $15.13 \pm 0.21^{\mathrm{a}, \mathrm{d}}$ & $\ldots$ & $\ldots$ & $\ldots$ \\
\hline & $J$ & $14.69 \pm 0.05^{\mathrm{a}}$ & $\ldots$ & $14.47 \pm 0.11^{\mathrm{b}}$ & $15.83 \pm 0.10^{\mathrm{c}}$ & $\ldots$ \\
\hline & $H$ & $14.21 \pm 0.02^{\mathrm{a}}$ & $\ldots$ & $13.97 \pm 0.08^{\mathrm{b}}$ & $14.65 \pm 0.08^{c}$ & $\ldots$ \\
\hline & $K_{s}$ & $\ldots$ & $\cdots$ & $13.96 \pm 0.12^{\mathrm{b}}$ & $14.12 \pm 0.05^{\mathrm{c}}$ & $\cdots$ \\
\hline & $M^{\prime}$ & $\cdots$ & $\cdots$ & $\cdots$ & $\cdots$ & $13.81 \pm 0.24^{\mathrm{a}}$ \\
\hline \multirow[t]{4}{*}{ HD $1160 \mathrm{C}$} & $Y$ & $13.74 \pm 0.02^{\mathrm{a}}$ & $\ldots$ & $\ldots$ & $\ldots$ & $\ldots$ \\
\hline & $J$ & $13.37 \pm 0.02^{\mathrm{a}}$ & $\ldots$ & $\ldots$ & $13.31 \pm 0.04^{\mathrm{c}}$ & $\ldots$ \\
\hline & $H$ & $12.81 \pm 0.02^{\mathrm{a}}$ & $\ldots$ & $\ldots$ & $12.54 \pm 0.03^{\mathrm{c}}$ & $\ldots$ \\
\hline & $K_{s}$ & & $\ldots$ & $\ldots$ & $12.18 \pm 0.06^{\mathrm{c}}$ & $\ldots$ \\
\hline
\end{tabular}

Notes. Photometry.

a This work.

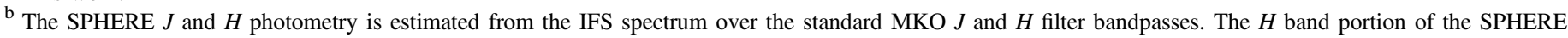

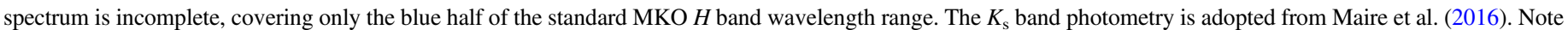
that we also adopt their $L^{\prime}$ measurement of $m\left(L^{\prime}\right)=13.60 \pm 0.10$ for HD $1160 \mathrm{~B}$.

c Nielsen et al. (2012).

d Note that the GPI $Y$ band filter extends to slightly longer wavelengths than HiCIAO.

signal. For simplicity, we analyze only the halo-subtracted images which lack companion signal loss.

Our SCExAO photometry of HD $1160 \mathrm{~B}$ are shown in the left columns of Table 2: $m(Y, J, H)=15.30 \pm 0.04,14.69 \pm 0.05$, and $14.21 \pm 0.02$. For HD $1160 \mathrm{C}$, we derive $m(Y, J$, $H)=13.74 \pm 0.02, \quad 13.37 \pm 0.02, \quad$ and $12.81 \pm 0.02$. Our errors consider (in quadrature) the $\mathrm{S} / \mathrm{N}$ of the detection (computed as in Currie et al. 2015), uncertainties in the primary magnitude, and a minor contribution of uncertainties in the attenuation of the neutral density filter.

We used aperture photometry to measure the brightness of HD $1160 \mathrm{~A}$ (in unsaturated images obtained with the neutral density filter) and HD 1160 BC. For the fainter HD $1160 \mathrm{~B}$ companion, we derived the contrast between it and the primary using a range of aperture diameters comparable to the FWHM of a point source in each passband. For the contrast of the brighter, wider-separation HD $1160 \mathrm{C}$ component, we used larger apertures enclosing much more $(>90 \%)$ of the primary and companion signals to guard against flux loss due to PSF degradation (e.g., vignetting of the field).

Our SCExAO HD $1160 \mathrm{~B} J$ and $H$ photometry shows good agreement with SPHERE measurements (Table 2, third column). SPHERE's brightness estimates are systematically brighter by $1.8 \sigma(0.22 \pm 0.12 \mathrm{mag})$ at $J$ band and $\approx 2.8 \sigma$ $(0.23 \pm 0.08 \mathrm{mag})$ at $H$ band. A good portion of the discrepancy at $H$ band is due to SPHERE's spectrum only covering half the $H$ band filter. Small differences at $J$ band may be due to SPHERE's flux calibration. SPHERE's spectrophotometry had to be corrected for signal loss due to aggressive processing; while the HiCIAO neutral density filter is flat across the bandpass, SPHERE's exhibits a slight wavelengthdependent attenuation at $J$ band.

In contrast, our photometry strongly disagrees with the measurements of $\mathrm{N} 12$ by $1.14 \mathrm{mag}$ at $J$ and 0.44 at $H$ (Table 2, columns 3 and 6). ${ }^{39}$ Similar discrepancies between NICI

\footnotetext{
39 For another estimate of HD 1160 B's $J$ band photometry, we downloaded and reduced archival $J$ band Keck/NIRC2 data for HD 1160 from 2004 July 14 (P.I. I. Song) using image processing methods identical to those described in this work. Our Keck detection has a substantially lower S/N than SCExAO and SPHERE. However, it agrees with the SCExAO and SPHERE photometry against the NICI photometry: $m_{J}=14.54 \pm 0.24$.
}

photometry and other instruments (Boccaletti et al. 2013) have been observed previously. The difference may be due to inaccurate flux normalization, possibly from mismeasured attenuation through the coronagraphic mask, or an inaccurate subtraction of the sky background annulus surrounding the companion (as was the case for early photometry of ROXs 42B"c," see Currie et al. 2014b).

\subsection{GPI Y Band Low-resolution Spectroscopy}

\subsubsection{Observations and Basic Processing}

HD 1160 B was observed with the GPI (Macintosh et al. 2006, 2014) in the $Y$ band $(0.957-1.120 \mu \mathrm{m}, R \approx 37$ ) on 2013 November 17 in $\sim 1^{\prime \prime}$ seeing by the GPI Verification and Commissioning team (Table 1). The observations consist of nine $88.7 \mathrm{~s}$ exposures in IFS mode (Larkin et al. 2014) using GPI's apodized pupil Lyot coronagraph with a pixel scale of $14.14 \pm 0.01$ mas pixel $^{-1}$ (Konopacky et al. 2014). As shown in Figure 2, the GPI $Y$ band filter extends $0.1 \mu \mathrm{m}$ past typical $Y$ band filters (see the appendix of Liu et al. 2012). It also extends blueward of the SPHERE $Y$ band data from M16, thus expanding the wavelength range at which HD $1160 \mathrm{~B}$ can be detected and analyzed beyond that offered by either SPHERE or SCExAO observations. The observations were obtained in ADI mode. However, like the SCExAO observations, the GPI sequence covered little parallactic motion $(\Delta$ P.A. $\sim 4.3)$.

We processed the images using the GPI data reduction pipeline version 1.3.0 (Maire et al. 2010, 2012; Perrin et al. 2014). The pipeline requires the location and spectral solution for every lenslet on the HAWAII-2RG detector. These lenslet locations were determined by using a cross-correlation between the deep argon calibration source images available on the GPI public webpage. ${ }^{40}$ The elevation of the telescope differed between the images of HD $1160 \mathrm{~B}$ and the daytime argon calibration lamp sequence. We calculated a shift to determine the overall change of the wavelength solution between the daytime calibrations and that appropriate for the observations of HD 1160 B.

\footnotetext{
${ }^{40}$ http://www.gemini.edu/sciops/instruments/gpi/public-data
} 


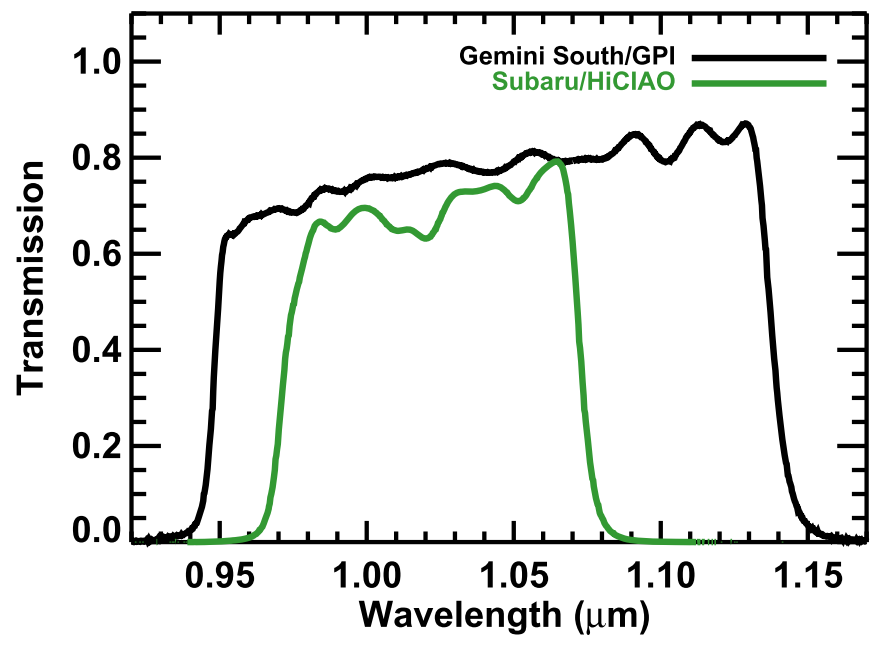

Figure 2. A comparison of the GPI $Y$ band filter and the HiCIAO $Y$ band filter. The GPI filter is slightly wider on both the blue and red end in comparison to the HiCIAO filter. This leads to slightly brighter photometry for the GPI $Y$ band as compared to our SCExAO/HiCIAO photometry for HD $1160 \mathrm{~B}$.

We further used the GPI data reduction pipeline to apply dark corrections, remove bad pixels, track satellite spot locations, convert each microspectrum into a 37 channel spectral cube $(0.957-1.120 \mu \mathrm{m})$, correct for distortion using the publicly available distortion solution (see footnote 38 ), and correct for atmospheric differential refraction. Each of the nine individual raw observations were processed in an identical way. HD $1160 \mathrm{~B}$ is visible in individual spectral channels of each data cube (Figure 3).

\subsubsection{Extraction of $H D 1160$ B Spectrum and Calibration}

The final, distortion-corrected, de-rotated, time-averaged data cube of HD 1160 has a smooth, slowly varying halo at HD 1160 B's angular separation (left panel, Figure 3). We removed this slow-varying background using a median highpass filter (right panel, Figure 3 ) with a box size of 11 pixels $(\approx 5 \lambda / D)$. We manually confirmed that this filtering did not result in a measurable loss in signal. Our final data cube had an $\mathrm{S} / \mathrm{N} \sim 90$ detection for HD $1160 \mathrm{~B}$ in the wavelengthcollapsed cube and $\mathrm{S} / \mathrm{N}>10$ for each channel.

To flux-calibrate our final data cube and extract HD 1160 B's flux-calibrated spectrum, we again followed steps outlined in the GPI data reduction pipeline. First, we extracted the fluxcalibrated spectra of the four satellite spots using the laboratory measured satellite-to-star flux ratio of $2.345 \times 10^{-4}$ from Wang et al. (2014) and assumed an A0V template spectrum from the Pickles stellar library (Pickles 1998) to characterize the host star spectrum of HD $1160 \mathrm{~A}$ (N12). ${ }^{41} \mathrm{We}$ adopted a 2-pixel aperture radius, a 5-20 pixel annulus to define the sky background, and the standard deviation of the four satellite spot flux density measurements as the uncertainty in absolute calibration in each spectral channel. ${ }^{42}$

To extract the spectrum of HD $1160 \mathrm{~B}$, we used the same aperture radius and background annulus used to extract the satellite spot spectra. The errors in the extracted and flux-

\footnotetext{
${ }^{41} \mathrm{HD} 1160 \mathrm{~A}$ is a well-known IR photometric standard star (Elias et al. 1982).

42 The upper-left spot spectrum contributed the most to the uncertainty in each spectral channel and in the wavelength-collapsed image, which is $\sim 20 \%$ fainter than the average of the other three spots.
}

calibrated HD 1160 B spectrum draw from the uncertainty in the spot calibration and the uncertainty in the background annulus signal surrounding HD $1160 \mathrm{~B}^{43} \mathrm{We}$ trimmed the HD $1160 \mathrm{~B}$ spectrum of the first and last few channels $(<0.953 \mu \mathrm{m}$ and $>1.12 \mu \mathrm{m})$, which had absolute calibration uncertainties $\gtrsim 10 \%$. The resulting flux calibrated spectrum spanning $0.957-1.120 \mu \mathrm{m}$ is shown in Figure 4. From the wavelength-collapsed data cube, we estimate a $Y$ band magnitude of $m(Y)=15.13 \pm 0.21$, consistent with our more precise SCExAO measurements (Table 2, third column).

\subsection{Rereduced Keck/NIRC2 $M^{\prime}$ Photometry}

M16 found significant discrepancies between their SPHERE/IRDIS photometry and that from N12 in the $J$ and $H$ passbands. Additionally, even after adopting the revised HD 1160 B NaCo $L^{\prime}$ photometry from M16 ( $\sim 0.2$ mag fainter), the implied $L^{\prime}-M^{\prime}$ color is $\sim-0.7$, characteristic of $L / T$ transition objects but far too blue for a mid-M dwarf like HD 1160 B (see Galicher et al. 2011).

Therefore, we re-reduced the same archival Keck/NIRC2 $M^{\prime}$ data first analyzed by N12 (HD $1160 \mathrm{C}$ was outside of the field of view). Basic processing followed standard steps we have previously used for thermal IR data (e.g., Currie et al. 2011) but without PSF subtraction. Briefly, after applying a linearity correction to the data, we constructed a sky frame for a given image from the five nearest (in time) images where the star is in a different dither position. After sky subtraction, we corrected for distortion, registered each image to a common center, subtracted off the radial intensity profile for each image, averaged the set of profile-subtracted images using 3- $\sigma$ outlier rejection, and applied a $5 \lambda / \mathrm{D}$ moving-box median filter to the combined image. As the combined image shows some residuals of the PSF halo, we computed the $\mathrm{S} / \mathrm{N}$ of the detection in a conservative fashion (not assuming that the image at HD 1160 B's location is photon noise dominated) as we have typically done with high-contrast imaging data sets (e.g., Currie et al. 2011).

Our re-reduction yields a $\mathrm{S} / \mathrm{N} \sim 4.6$ detection of $\mathrm{HD} 1160 \mathrm{~B}$ with a measured apparent magnitude of $\mathrm{m}\left(M^{\prime}\right)=13.81 \pm 0.24$ (Table 2, far-right column), where the intrinsic $\mathrm{S} / \mathrm{N}$ of the detection contributes almost all of the photometric uncertainty. HD $1160 \mathrm{~B}$ is about $63 \%$ brighter at $M^{\prime}$ than reported in N12. The implied $L^{\prime}-M^{\prime}$ color of $\sim-0.21 \pm 0.23$ is in agreement with expected colors for mid-M to early $\mathrm{L}$ objects (Galicher et al. 2011).

\section{CHARACTERIZATION OF HD 1160 B AND C}

\subsection{Analysis of the HD $1160 \mathrm{~B}$ and C Photometry}

To compare HD $1160 \mathrm{~B}$ and C to field MLT dwarfs, we adopt our SCExAO $Y J H$ photometry. We assume a distance of $103.1 \pm 5.3 \mathrm{pc}$ (van Leeuwen 2007), yielding absolute magnitudes of $Y=10.23 \pm 0.11, \quad J=9.63 \pm 0.10$ and $H=9.14 \pm$ 0.10 mag. Figure 5 compares our HD $1160 \mathrm{~B}$ photometry to the sequence of MLT dwarfs from Dupuy \& Liu (2012), and M-dwarf standards of Kirkpatrick et al. (2010) and references therein. We plot $Y J H$ colors of $J-H=0.48 \pm 0.05, Y-J=0.61 \pm$

\footnotetext{
$\overline{43}$ For a separate spectral extraction independent of the GPI data reduction pipeline, we performed aperture photometry using (aper.pro) at the location of the companion, for each wavelength slice, assuming the background to be zero. The two methods show strong agreement, to within the calculated errors/ channel.
} 

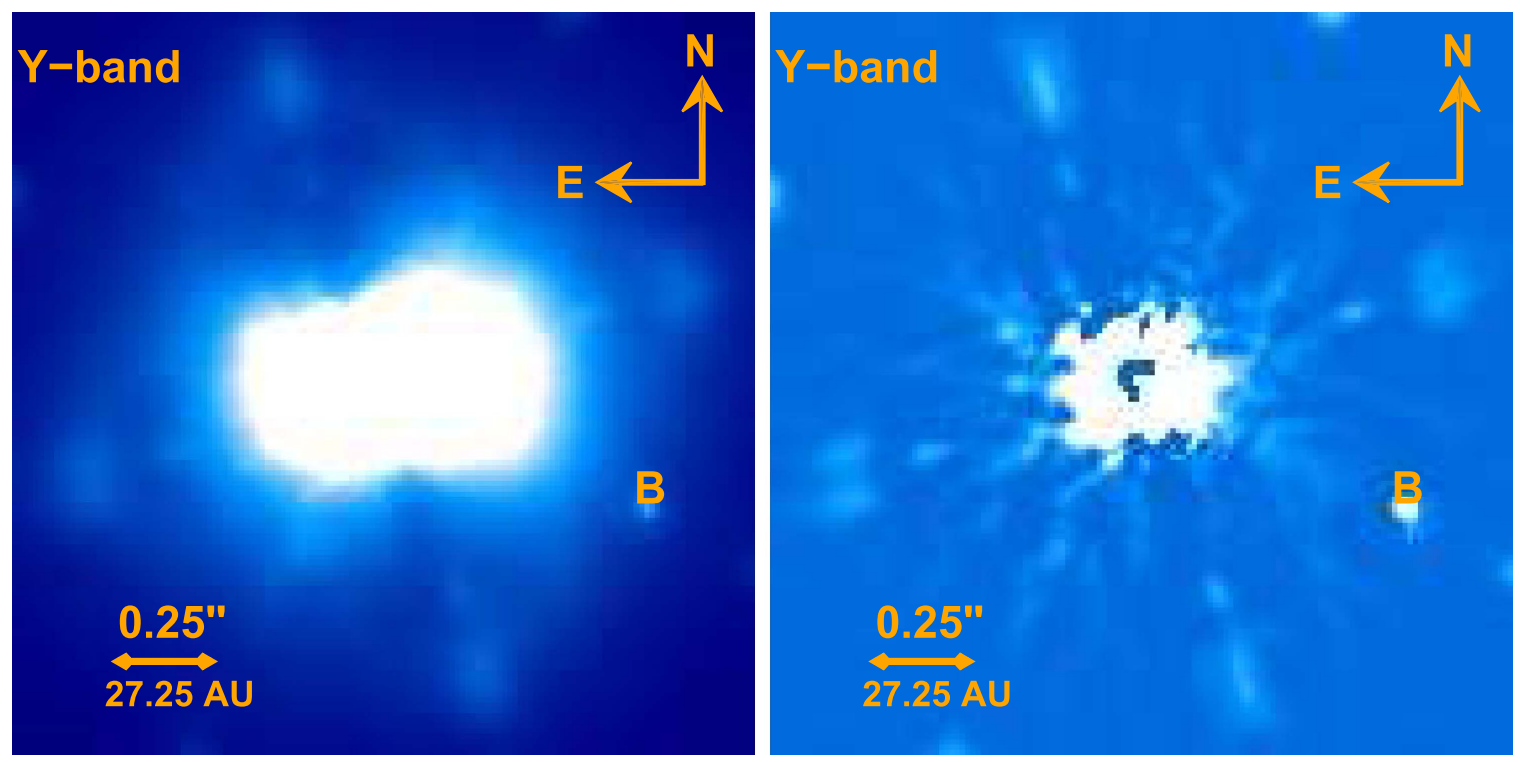

Figure 3. The wavelength-collapsed, de-rotated, stack of temporal frames for Gemini Planet Imager $Y$ band observations of HD $1160 \mathrm{~B}$. To remove the slowly varying background due to the smooth speckle halo, the original image (left) is high-pass filtered (right) using a median with a box size of 11 pixels $(\sim 5 \lambda / D)$.

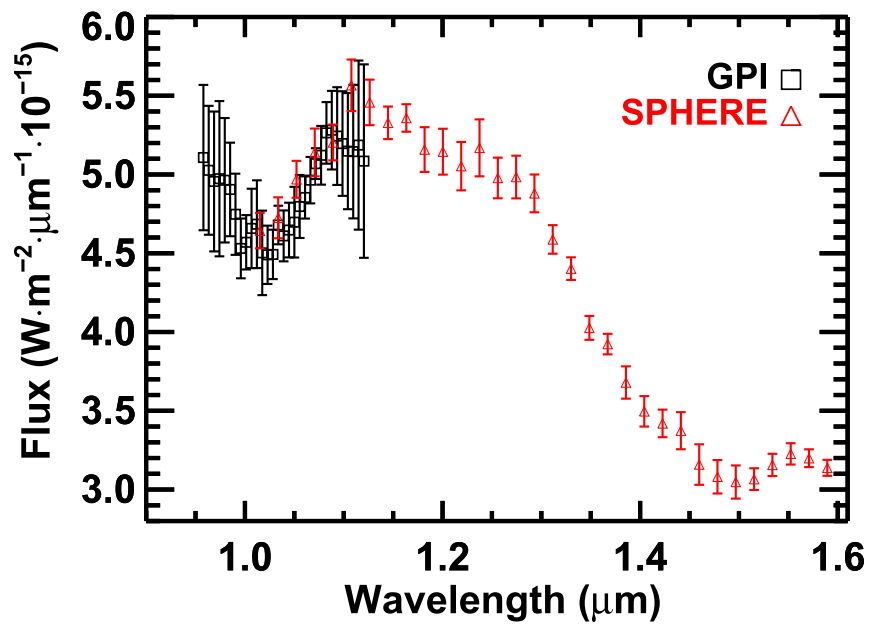

Figure 4. Comparison between the GPI $Y$ band spectrum of HD $1160 \mathrm{~B}$ $(0.957-1.120 \mu \mathrm{m}$, black squares) and the SPHERE spectrum (red triangles) covering $1.0-1.6 \mu \mathrm{m}$. There is good agreement between the SPHERE spectra and our higher-resolution GPI spectrum (see Section 2.2.2) within errors, although the SPHERE spectra are slightly brighter over the $Y$ bandpass. The GPI spectra errors are larger due to the higher resolution (the wavelength bins are $\sim 5.4 \mathrm{~nm}$ for GPI and $\sim 18.5 \mathrm{~nm}$ for SPHERE). We use a combined GPI and SPHERE spectrum for our atmosphere modeling (see 3.3).

0.06 colors and the absolute magnitudes in $Y, J$, passbands for HD $1160 \mathrm{~B}$ as the blue star in Figure 5. In all cases, HD 1160 B's photometric points are consistent with the field dwarf sequence. We find HD 1160 B's colors in agreement with the M5-M6 spectral standards of Kirkpatrick et al. (2010) (transition of green and red diamonds in Figure 5). Similarly, as shown by the pink star in Figure 5, we find the NIR colors and the $Y J H$ absolute magnitudes of HD $1160 \mathrm{C}$ in agreement with M3-M4 spectral standards of Kirkpatrick et al. (2010).

As a further check, we compare HD 1160 B's nominal colors to those if we adopt photometry extracted from the SPHERE spectrum in $J H$ (Table 2) and photometry from partial coverage in $Y$ band $(m(Y)=15.19 \pm 0.02 ; M(Y)=10.12 \pm 0.02)$. The SPHERE-derived photometry is slightly redder in $Y-J$ but otherwise suggests that HD $1160 \mathrm{~B}$ is consistent with the field sequence (pink and blue triangles, Figure 5).

\subsection{Analysis of the HD 1160 B Spectrum}

To compare HD 1160 B's full NIR spectrum to that from other objects, we constructed a merged $Y J H$ spectrum from GPI and SPHERE data. We applied an offset to the GPI spectrum such that its photometry integrated over the $Y$ band perfectly matched the SCExAO $Y$ band photometry and a separate offset to the SPHERE spectrum such that its bandintegrated photometry matched that of SCExAO's as well. We adopted the GPI (SPHERE) measurements shortwars (longward) of $1.12 \mu \mathrm{m}$.

As implied by N12 and M16 and shown in Section 4 of this paper, HD 1160's age is likely intermediate between that of low-mass members of young star-forming regions such as Upper Sco (11 \pm 2 Myr, Pecaut et al. 2012) and older field objects ( $>1$ Gyr). For spectral typing HD 1160 B, we therefore compare the GPI+SPHERE spectrum to field M-dwarf spectral standards from the SPEX library (Rayner et al. 2003) and Upper Sco members (Dawson et al. 2014).

We perform the spectral typing by binning the template NIR spectra to the resolution of the GPI and SPHERE spectra and computing the $\chi^{2}$ as:

$$
\chi^{2}=\sum_{i=1}^{i=57} \frac{\left(F_{\mathrm{i}, \text { obs }}-F_{\mathrm{i}, \text { template }}\right)^{2}}{\sigma_{\mathrm{i}, \mathrm{obs}}^{2}+\sigma_{\mathrm{i}, \text { template }}^{2}},
$$

where the flux $F$ is in watts $\mathrm{m}^{-2} \mu \mathrm{m}^{-1}$ and index $i$ corresponds to one of the 57 spectral channels of the flux calibrated GPI and SPHERE spectra. We weighted the GPI and SPHERE spectra equally.

As shown in Figure 6, the GPI and SPHERE spectrum shape is poorly reproduced for Upper Sco M8 member 2M16101 -28563 for spectral channels $<1.0 \mu \mathrm{m}$ and $1.3-1.4 \mu \mathrm{m}$. The disagreement is greater for L0.8 Upper Sco member 2M16195 -28322 (top of Figure 6). On the other hand, M5.2-M6.8 Upper Sco members have $\Delta \chi^{2}$ within the $95 \%$ confidence interval for 57 degrees of freedom (see Press et al. 1992). We 

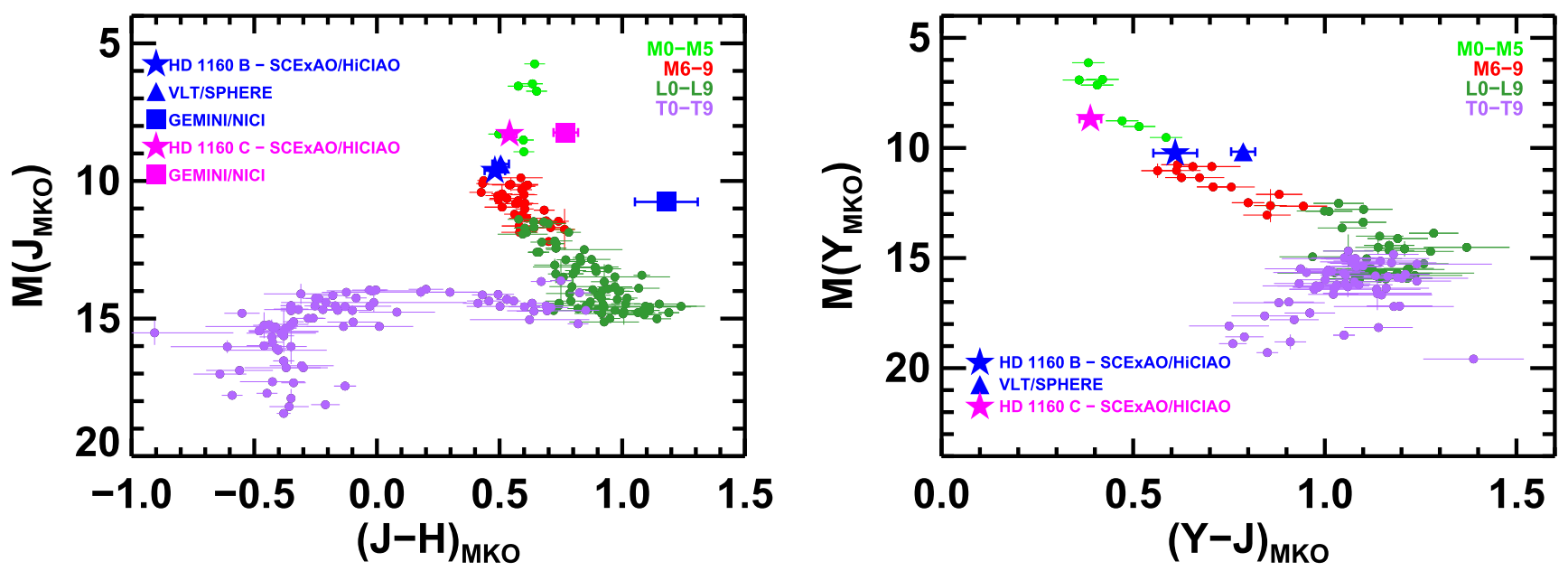

Figure 5. Color-magnitude diagrams comparing SCExAO/HiCIAO (this work), Gemini/NICI photometry (Nielsen et al. 2012), and the integrated-spectra photometry of SPHERE observations (Maire et al. 2016). The M3-M6 main sequence is from dwarf standards of Kirkpatrick et al. (2010). The M6-T9 main-sequence dwarfs that form the color-magnitude sequence is adopted from Dupuy \& Liu (2012). At $Y$ are the Dupuy \& Liu (2012) integrated spectra for the M6-T9 dwarfs to compute the photometry using the UKIRT/WFCAM $Y$ band (Hewett et al. 2006) .

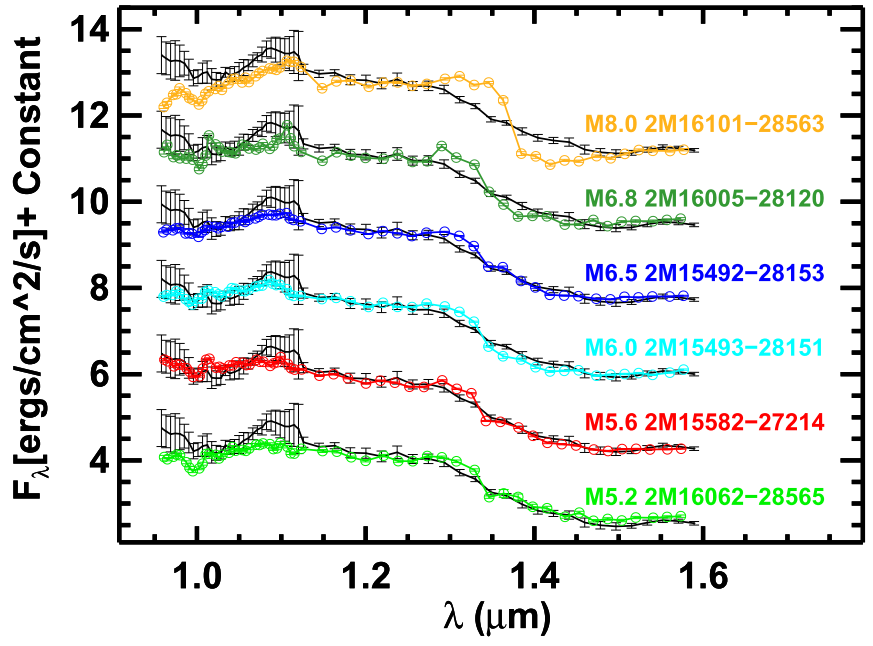

Figure 6. The combined SPHERE and GPI spectra of HD 1160 B are best reproduced by the SPEX spectra of M5.2-M6.8 members of Upper Sco from Dawson et al. (2014). M8 and later members of Upper Sco do not reproduce the semi-smooth slope of the SPHERE spectrum from 1.2 to $1.6 \mu \mathrm{m}$. The SPEX spectra were binned to the resolution of the GPI and SPHERE respectively, and scaled to the corresponding flux levels to minimize the $\chi^{2}$. Given that the GPI spectrum is higher resolution and the SPHERE spectrum has greater wavelength coverage, we weighted both equally in our spectral typing procedure (see Section 3.2). $F_{\lambda}$ is normalized by a factor of $10^{-15}$. The constant is an an offset in unit steps of $1.7 \times 10^{-15} \mathrm{erg} \mathrm{cm}^{-2} \mathrm{~s}^{-1}$.

therefore find the spectral type of HD $1160 \mathrm{~B}$ as compared to Upper Sco Members to be $\mathrm{M} 6_{-0.8}^{+0.8}$.

Similarly, Figure 7 compares the GPI and SPHERE spectra of HD 1160 B to M2-M8 field SPEX spectra standards from Kirkpatrick et al. (2010). We find that the overall spectral shape is best reproduced by M5 standard 2M01532+36314 or M6 standard 2M13272+09464. The overall shape of the spectrum is poorly reproduced by the linear slope of M4 or earlier standards across $0.95-1.6 \mu \mathrm{m}$. Standard spectra later than M6 poorly reproduce the smooth portion of the HD $1160 \mathrm{~B}$ spectrum at $1.3-1.4 \mu \mathrm{m}$ as well as the slope at $<1.0 \mu \mathrm{m}$. Therefore, we find the spectral type of HD $1160 \mathrm{~B}$ as compared to field $M$ dwarfs to be M5. $5_{-0.5}^{+0.5}$, in agreement with the spectral types derived for HD 1160 B compared to Upper Sco members

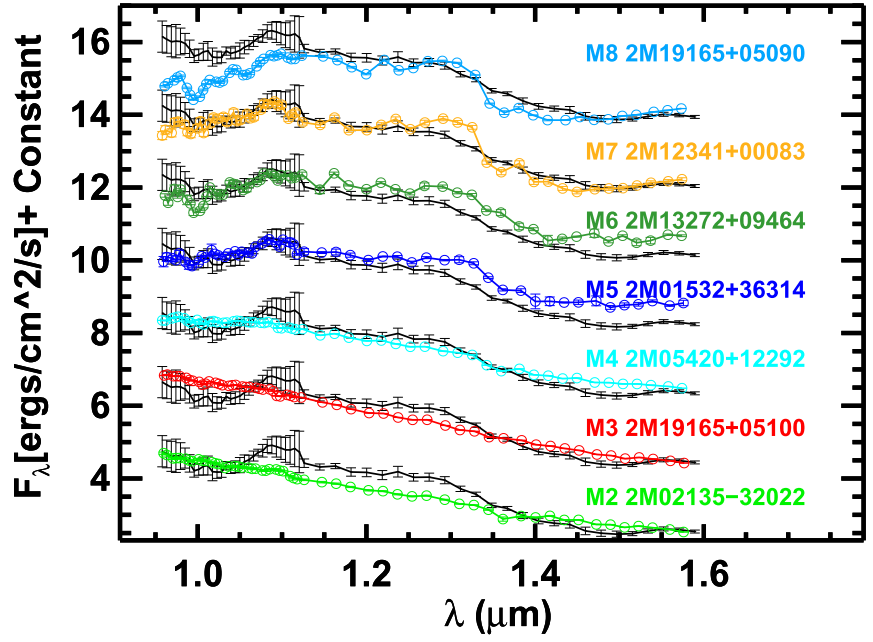

Figure 7. Similar to Figure 6, the combined SPHERE and GPI spectra of HD $1160 \mathrm{~B}$ are best reproduced by the SPEX spectra of M5-M6 field standards from Kirkpatrick et al. (2010) (see Figure 9). Standards M4 or earlier spectra do not reproduce the overall shape of the HD 1160 spectrum, while standards M7 and later also do not accurately produce the shape of the HD 1160 spectrum from 1.3 to $1.6 \mu \mathrm{m}$. The method of comparing standard spectral templates to the spectrum of HD 1160 is identical to Figure 6 and detailed in Section 3.2. $F_{\lambda}$ is normalizeded by a factor of $10^{-15}$. The constant is an an offset in unit steps of $1.9 \times 10^{-15} \mathrm{erg} \mathrm{cm}^{-2} \mathrm{~s}^{-1}$.

above. We adopt a spectral type for HD $1160 \mathrm{~B}$ of M5.5 $5_{-0.5}^{+1.0}$ as an average between the dwarf and Upper Sco spectral types, finding agreement with the M16 spectral type of M6 $6_{-0.5}^{+1.0}$.

\subsection{Atmospheric Modeling of HD $1160 \mathrm{~B}$}

Atmosphere Models Considered. To explore HD 1160 B's atmospheric properties, we compare the objects broadband photometry and $Y J H$ GPI+SPHERE spectrum to predictions from planet atmosphere models, adopting a range of effective temperatures and surface gravities. We consider the BT-Settl 2013 models of Allard et al. (2012) with temperatures of 2500-3500 K, surface gravities of $\log g=3.5-5.5 \mathrm{dex}$ and solar metallicity. Using the same atmosphere models, M16 concluded that spectral energy distribution (SED) modeling 
Table 3

Model Fitting Results

\begin{tabular}{lcc}
\hline \hline & Photometry & Spectroscopy \\
\hline Wavelength Range & $Y J H K_{s} L^{\prime} M^{\prime}$ & $0.95-1.6 \mu \mathrm{m}$ \\
$T_{\text {eff }} \mathrm{K}(95 \%$ Confidence) & $2900-3500$ & $3000-3100 \mathrm{~K}$ \\
$\log g$ dex (95\% Confidence) & $3.5-5.5$ & $2900 \mathrm{~K}($ for $\log g=4.5)$ \\
& $4.0-4.5$ & 5.0 (for $3000 \mathrm{~K})$ \\
Models fitting both & $T_{\text {eff }}=3000-3100, \log g=4.0-4.5$ & \\
Photometry and Spectra: & $T_{\text {eff }}=2900 \mathrm{~K}, \log g=4.5 ; T_{\text {eff }}=3000 \mathrm{~K}, \log g=5.0$ & \\
\hline
\end{tabular}

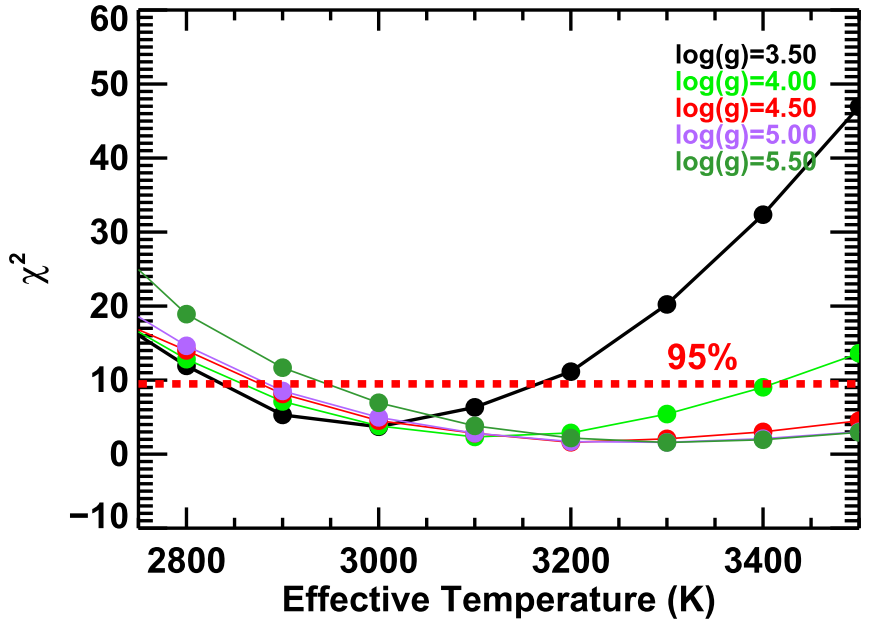

Figure 8. We find the photometry unable to constrain HD 1160 B's effective temperature to better than $T_{\text {eff }}=2900-3500 \mathrm{~K}$ and surface gravity $\log g$ to $3.5-5.5$ dex. $\chi^{2}$ distributions for fitting HD $1160 \mathrm{~B}$ photometry as a function effective temperature and surface gravity. The horizontal dashed red line corresponds to the $95 \%(2 \sigma)$ confidence limit, identifying acceptably fitting models. Data points (circles) below this line represent BT-settl models that are in good agreement with the photometry of HD $1160 \mathrm{~B}$.

favors a subsolar metallicity for HD $1160 \mathrm{~B}$. Using revised/ new photometry and spectroscopy, we assess whether or not HD 1160 B may yet have a solar metallicity.

Fitting Method. Following previous approaches (e.g., Currie et al. 2014b), we fit our $Y J H$ (SCExAO, this work) $K_{s}$ (SPHERE, M16) $L^{\prime}$ (NaCo, M16) $M^{\prime}$ (Keck, this work) photometry and $Y J H$ GPI+SPHERE spectroscopy. To model the photometry, we compare the measured flux densities with predicted ones from the model spectra convolved with the MKO filter functions. The BT-Settl grid has significantly higher spectral resolution than the data $(R \approx 37, \mathrm{~S} / \mathrm{N} \gg 10$ per channel). Thus, to model the spectrum of HD $1160 \mathrm{~B}$, we rebin the BT-Settl spectra to the resolution of our extracted spectrum. We treat the planet radius as a free parameter, varying it from $R$ $\sim 0.75 R_{\mathrm{J}}$ to $R \sim 2.25 R_{\mathrm{J}}$, which spans the wide range in radii predicted from the models (Baraffe et al. 2003).

We identify the set of BT-Settl models consistent with the spectra or photometry at the $95 \%$ confidence limits given the number of degrees of freedom. We adopt the measured photometric error, listed in Table 2, for model fitting. We incorporate our distance uncertainty of $\pm 5.3 \mathrm{pc}$ (van Leeuwen 2007) in the uncertainty estimate for the planet radius. We do not fit for $T_{\text {eff }}$ and $\log g$ parameters via $\chi^{2}$ minimization; rather, we compare the $\chi^{2}$ to a grid of atmosphere models with pre-determined $T_{\text {eff }}$ and $\log g$, treating the radius as a scaling parameter. Given six photometric points and four degrees of freedom, the $95 \%$ cutoff is $\chi_{\lim }^{2}=9.49$. Given 57

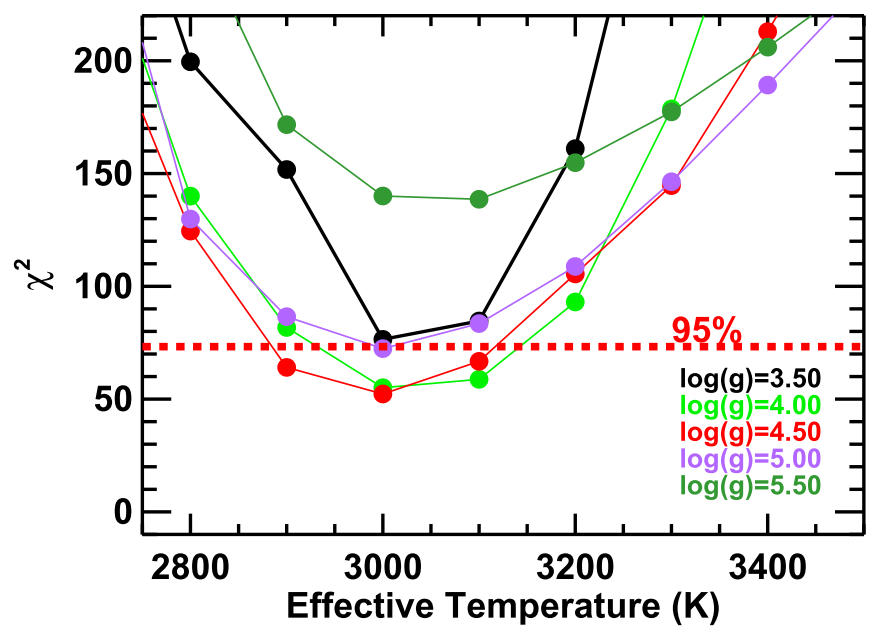

Figure 9. Same as Figure 8 except for the spectroscopy. With the $0.95-1.6 \mu \mathrm{m}$ spectroscopy we find the best fits for an effective temperature $T_{\text {eff }}=3000-3100 \mathrm{~K}$ and surface gravity $\log (g)=4.0-4.5 \mathrm{dex}$, while $T_{\text {eff }}=2900 \mathrm{~K}, \log g=4.5$ and $T_{\text {eff }}=3000 \mathrm{~K}, \log g=5.0$ are also marginally acceptable.

spectral channels and 55 degrees of freedom, models with $\chi^{2} \leqslant 73.3$ are consistent with the data.

Photometry and Spectroscopy Fitting Results. Table 3 summarizes our model-fitting results to the $Y J H K_{s} L^{\prime} M^{\prime}$ photometry and $0.95-1.6 \mu \mathrm{m}$ GPI+SPHERE spectroscopy. ${ }^{44}$ Figures 8 and 9 display the $\chi^{2}$ distribution for our model fits to the photometry and spectroscopy, respectively. Figure 10 displays an acceptably fitting model compared to the $Y J H K_{s} L^{\prime} M^{\prime}$ photometry and 0.95-1.6 $\mu \mathrm{m}$ spectroscopy.

For fitting the photometry alone, our best-fit model has a reduced chi-square $\chi_{\text {red }}^{2}=0.63$ (d.o.f $=6-2=4$ ). Our $95 \%$ cutoff for photometry fits corresponds to surface gravities of $\log g=3.5-5.5 \mathrm{dex}$, and effective temperature of $T_{\text {eff }}=2900-3500 \mathrm{~K}$. Our model fits to the photometry are rather degenerate given our small number (six) of photometry points and the similarity of broadband colors for objects at $T_{\text {eff }}=2900-3500 \mathrm{~K}$.

However, combining fits to the photometry and spectroscopy significantly constrains the temperature and surface gravity of HD 1160 B. Our best-fit model for the spectrum has a reduced chi-square $\chi_{\text {red }}^{2}=0.92($ d.o.f $=57-2=55)$ with a surface gravity of $\log g=4.5 \mathrm{dex}$ and effective temperature of $T_{\text {eff }}=3000 \mathrm{~K}$. The solutions generally agree with M16 and cluster around $T_{\text {eff }}=3000-3100 \mathrm{~K}$ and $\log g=4-4.5$, although we cannot rule out a $2900 \mathrm{~K}, \log g=4.5$ model nor

\footnotetext{
${ }^{44}$ The SPHERE and NICI photometry at $K_{\mathrm{s}}$ agree to within errors. As a separate check on these results, we downloaded and reduced $K_{\mathrm{S}}$ data from the Keck/NIRC2 archive and derived photometry that agreed with both measurements, within errors.
} 

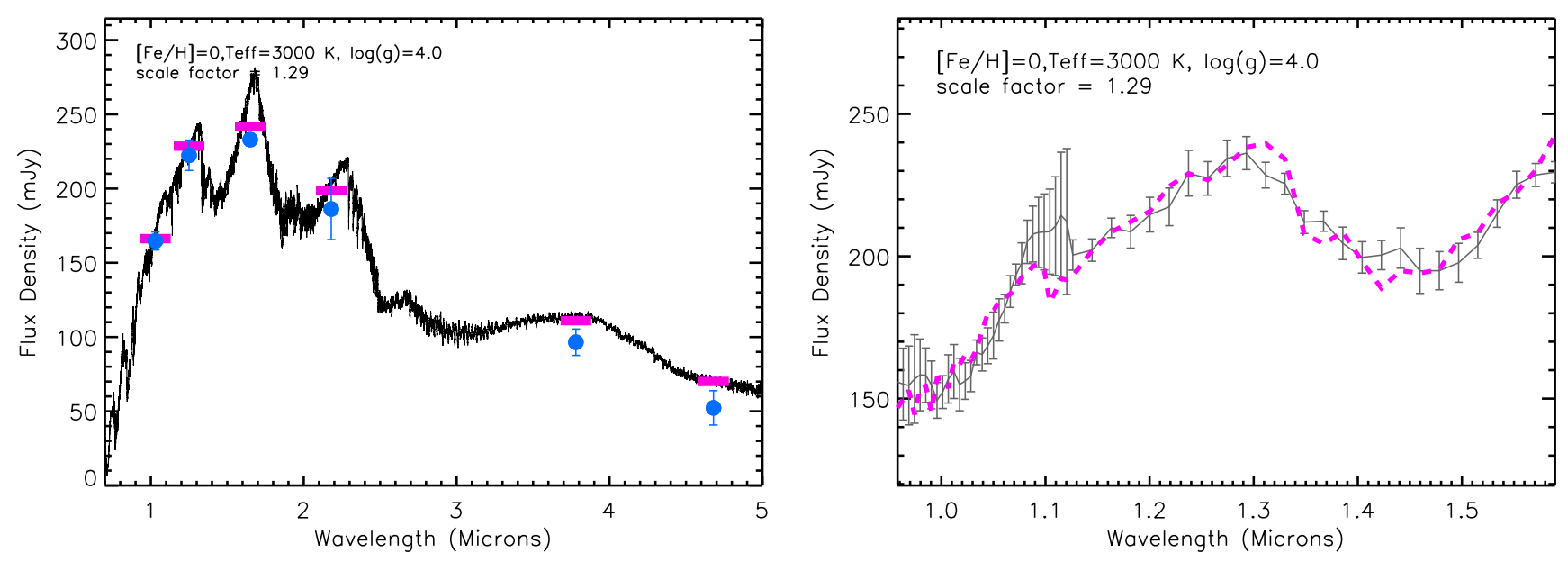

Figure 10. Example model fitting both the photometry (left) and spectroscopy (right) of HD $1160 \mathrm{~B}: T_{\text {eff }}=3000 \mathrm{~K}, \log g=4.0$. The photometric points are denoted by gray dots. In the left panel, the model is depicted as a thick black line and horizontal magenta lines show the predicted photometry from the model in photometric bandpasses. In the right panel, the spectrum is denoted by a gray line, and the model is depicted as a magenta dashed line.

a $3000 \mathrm{~K}, \log g=5.0$ model. We identify a suite of acceptably fitting models with a solar metallicity, not a subsolar metallicity. Thus, subsolar metallicities are not required to reproduce HD 1160 B's spectrophotometry.

\subsection{Inferred Properties of HD 1160 B from Best-fitting Models}

The BT-Settl models matching HD 1160 B's photometry and spectroscopy allow us to infer a radius for the object. Compared to the default radius we used $\left(1.2 R_{J}\right)$, our fits require radii larger by factors of $1.22-1.37 \times$ and $0.98-1.42 \times$ for the spectra and photometry respectively. The set of parameter space fitting photometry and spectra simultaneously covers $R=1.55 \pm$ $0.10 R_{\mathrm{J}}$ using our model fits to the HD $1160 \mathrm{~B}$ GPI+SPHERE spectrum, where errors in scaling the radius of our atmosphere models include the distance uncertainty error of $\approx 5 \%$. HD 1160 B's luminosity, from our best-fit effective temperature and radius from the spectroscopy, is $\log L / L_{\odot}=-2.76 \pm 0.05 \mathrm{dex}$, in good agreement with $\log L / L_{\odot}=-2.81 \pm 0.10 \mathrm{dex}$ for $\mathrm{HD}$ 1160 B from M16.

\subsection{Physical Characteristics of HD $1160 \mathrm{C}$}

We adopt an effective temperature of $T_{\text {eff }}=3260 \pm 100$ for HD $1160 \mathrm{C}$ using the M3.5 $\pm 0.5 \mathrm{SPEX} / \mathrm{IRTF}$ spectral type from N12. We obtain this $T_{\text {eff }}$ by averaging the $T_{\text {eff }}$ of an M3 and M4 5-30 Myr star from the relations of Pecaut \& Mamajek (2013) (see their Table 5). Given that HD $1160 \mathrm{C}$ is likely not 5-30 Myr, the spectral type- $T_{\text {eff }}$ relations in Table 5 of Pecaut \& Mamajek (2013) may not apply. The spectral type- $T_{\text {eff }}$ relations for 50-300 Myr M-dwarfs (like HD 1160 C) are expected to lie in between the relations for young stars and field stars. As an additional check, using the spectral type- $T_{\text {eff }}$ relations for field stars from Pecaut \& Mamajek (2013) gives an effective temperature of $T_{\text {eff }}=3300 \pm 100$ for HD $1160 \mathrm{C}$, which is within the error of our adopted $T_{\text {eff }}$.

We use our $J$ band absolute magnitude of $8.29 \pm 0.10 \mathrm{mag}$ and a bolometric correction of $\mathrm{BC}_{\mathrm{J}}=1.875 \pm 0.035$ from the relations of Pecaut \& Mamajek (2013) for young stars to estimate a bolometric magnitude of $10.16 \pm 0.11 \mathrm{mag}$. We calculate the bolometric correction $\mathrm{BC}_{\mathrm{J}}=1.875 \pm 0.035$ by averaging the bolometric corrections of a M3 and M4 star from Table 5 of Pecaut \& Mamajek (2013). We compute a corresponding luminosity of $\log L / L_{\odot}=-2.16 \pm 0.04$ dex, using a bolometric magnitude of 4.755 for the $\mathrm{Sun}^{45}$, in fair agreement with $\log L / L_{\odot}=-2.05 \pm 0.06$ dex from M16.

\section{ANALYSIS OF THE HD 1160 SYSTEM AGE AND METALLICITY}

\subsection{Motivation}

The age of the HD 1160 system is critical for interpreting the nature of the primary's two companions. Furthermore, HD 1160 provides a tight constraint on evolutionary models: the models should predict a common age given the observed effective temperatures and luminosities for three stars simultaneously. Given that HD $1160 \mathrm{~A}$ is on the main sequence, its age cannot be greater than the $300 \mathrm{Myr}$ main-sequence lifetime of an A0 star (e.g., Siess et al. 2000). N12 derive a system age of $10-100 \mathrm{Myr}$, while M16 derive a much wider age estimate of 30-300 Myr.

Here, we re-investigate the age of the system based on the derived $T_{\text {eff }}$ and $\log L / L_{\odot}$ for HD 1160 A, B and C. For stars close to the hydrogen burning limit and brown dwarfs just below it, recent results suggest difficulties in deriving ages and masses from HR diagram positions (Dupuy et al. 2009, 2010, 2015, 2016; Kraus et al. 2015), while ages derived from the upper main sequence are arguably preferable (Soderblom et al. 2014, p. 219). Thus, we report two possible age ranges: one considering HD $1160 \mathrm{~A}$ alone and one considering all three components jointly.

Our derived system parameters are detailed in Table 4 . We estimate the temperature and luminosity of HD $1160 \mathrm{~A}$ as follows. We fit the SED of HD 1160 A using the NextGen atmosphere models of Hauschildt et al. (1999). The photometry that we fit from the literature spans a wavelength range of $0.16-22 \mu \mathrm{m}$ (Figure 11 and Table 5). Our best-fit model atmosphere implies an effective temperature of $T_{\text {eff }}=9011 \pm 85 \mathrm{~K}$. The bolometric flux obtained by integrating the model SED provides an estimated $\log L / L_{\odot}=$ $1.12 \pm 0.07$.

\footnotetext{
$\overline{45}$ https://sites.google.com/site/mamajeksstarnotes/basic-astronomical-datafor-the-sun
} 
Table 4

Properties of the HD 1160 System

\begin{tabular}{|c|c|c|c|c|}
\hline Property & HD $1160 \mathrm{~A}$ & HD $1160 \mathrm{~B}$ & HD $1160 \mathrm{C}$ & Unit \\
\hline $\begin{array}{l}\text { R.A. (ep } \\
\text { J2000) }\end{array}$ & & $00: 15: 57.3025^{\mathrm{a}}$ & & \\
\hline $\begin{array}{l}\text { Decl. (ep } \\
\text { J2000) }\end{array}$ & & $+04: 15: 04.018^{\mathrm{a}}$ & & \\
\hline Distance & & $103.1 \pm 5.3^{\mathrm{a}}$ & & $\mathrm{pc}$ \\
\hline$\mu_{\alpha}$ & & $21.15 \pm 0.62^{\mathrm{a}}$ & & mas $\mathrm{yr}^{-1}$ \\
\hline$\mu_{\delta}$ & & $-14.20 \pm 0.24^{\mathrm{a}}$ & & mas $\mathrm{yr}^{-1}$ \\
\hline $\begin{array}{l}\text { Age, HD } \\
1160 \mathrm{~A}\end{array}$ & & $20-125$ & & Myr \\
\hline $\begin{array}{r}\text { Age, HD } \\
1160 \mathrm{~A}, \\
\text { B and C }\end{array}$ & & $80-125$ & & Myr \\
\hline$M_{Y}(\mathrm{MKO})$ & $1.89 \pm 0.10^{\mathrm{b}}$ & $10.23 \pm 0.11$ & $8.67 \pm 0.10$ & mag \\
\hline$M_{J}$ & $1.91 \pm 0.10$ & $9.63 \pm 0.10$ & $8.29 \pm 0.10$ & mag \\
\hline$M_{H}$ & $1.94 \pm 0.10$ & $9.14 \pm 0.10$ & $7.75 \pm 0.10$ & mag \\
\hline$M_{K s}$ & $1.97 \pm 0.10$ & $8.88 \pm 0.12^{\mathrm{c}}$ & $7.15 \pm 0.12^{\mathrm{d}}$ & mag \\
\hline $\begin{array}{l}\Delta Y \\
\quad(\mathrm{MKO})\end{array}$ & & $8.34 \pm 0.04$ & $6.78 \pm 0.01$ & mag \\
\hline$\Delta J$ & & $7.72 \pm 0.02$ & $6.38 \pm 0.01$ & mag \\
\hline$\Delta H$ & & $7.20 \pm 0.02$ & $5.80 \pm 0.01$ & mag \\
\hline$\Delta K$ & & $6.91 \pm 0.10^{c}$ & $5.14 \pm 0.06^{\mathrm{d}}$ & mag \\
\hline $\mathrm{SpT}$ & $\mathrm{A} 0^{\mathrm{d}}$ & M5. $5_{-0.5}^{+1.0}$ & $\mathrm{M} 3.5 \pm 0.5^{\mathrm{d}}$ & \\
\hline$T_{\text {eff }}$ & $9011 \pm 85$ & $3000-3100$ & $3260 \pm 100^{\mathrm{e}}$ & $\mathrm{K}$ \\
\hline $\log L / L_{\odot}$ & $1.12 \pm 0.07$ & $-2.76 \pm 0.05$ & $-2.16 \pm 0.04$ & dex \\
\hline $\log (g)$ & & $4.0-4.5$ & & dex \\
\hline \multirow[t]{3}{*}{ Mass } & $2.2^{\mathrm{d}}$ & & & $M_{\odot}$ \\
\hline & & $35-90^{\mathrm{f}}$ & $110-200^{\mathrm{f}}$ & $M_{\mathrm{J}}$ \\
\hline & & $70-90^{\mathrm{g}}$ & $175-200^{\mathrm{g}}$ & $M_{\mathrm{J}}$ \\
\hline
\end{tabular}

Notes. Properties of the HD 1160 system.

a van Leeuwen (2007).

${ }^{\mathrm{b}}$ Calculated from our SED fit to HD 1160 A photometry (see Section 4).

${ }^{\mathrm{c}}$ Calculated by Maire et al. (2016) at the $K_{s}$ passband using the SPHERE spectrum and IRDIS $K_{1}$ and $K_{2}$ photometry.

${ }^{\mathrm{d}}$ Nielsen et al. (2012).

${ }^{\mathrm{e}}$ Maire et al. (2016).

${ }^{\mathrm{f}}$ Mass ranges from Baraffe et al. (2015) models using an age derived for HD 1160 A alone (see Section 4).

${ }^{\mathrm{g}}$ Mass ranges from Baraffe et al. (2015) using an age derived for HD $1160 \mathrm{~A}$, $B$ and C (see Section 4).

\subsection{Methodology}

To derive the range of acceptable ages, we compare the system components' luminosities and temperatures to evolutionary models. For HD $1160 \mathrm{~A}$ and C, we consider the Dartmouth evolutionary models (Dotter et al. 2008; Feiden et al. 2011), the Yonsei-Yale ( $\left.\mathrm{Y}^{2}\right)$ models (Yi et al. 2003; Spada et al. 2013), and the MESA Isochrones and Stellar Tracks (MIST), which use the Modules for Experimental Astrophysics (Paxton et al. 2011, 2013; Choi et al. 2016; Dotter 2016). We also consider the Baraffe15 models (Baraffe et al. 2015) for HD $1160 \mathrm{~B}$ and $\mathrm{C}$. To arrive at a final age range for HD $1160 \mathrm{~A}$ and $\mathrm{C}$, we take the median of the ranges derived from each individual evolutionary model comparison.

All evolutionary models assume solar composition and metallicity. Our atmospheric modeling (Section 3.3) suggests that HD 1160 B's spectrophotometry is consistent with a solar metallicity atmosphere. Provided that other components of HD 1160 can match solar metallicity isochrones,

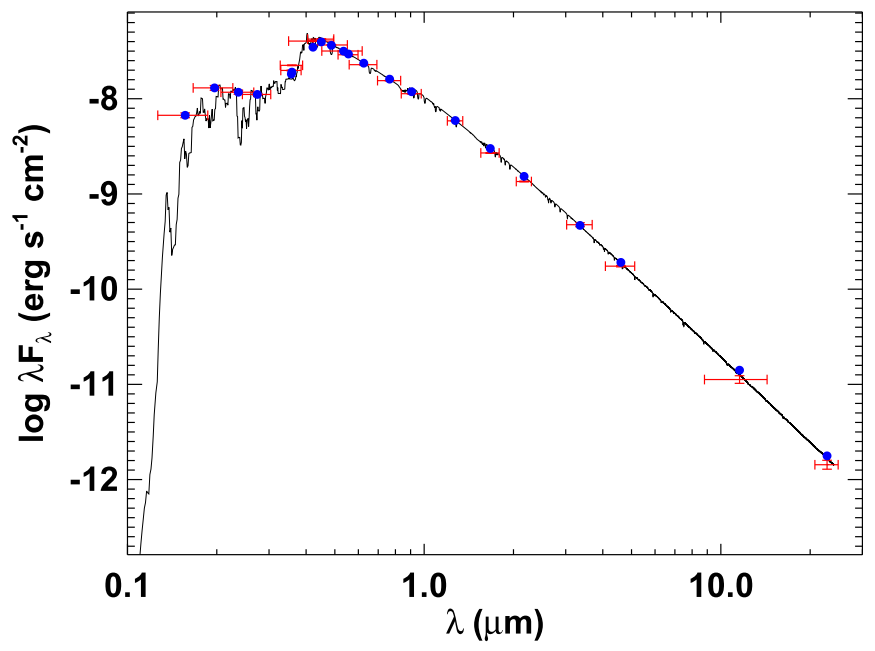

Figure 11. SED fit to catalog photometry spanning a wavelength range of 0.16-22 $\mu \mathrm{m}$ with a NextGen atmosphere model. Red symbols are the observed fluxes with errors (horizontal "error bars" are the photometric passband widths) and blue symbols are the model fluxes. We obtain a best-fit effective temperature of $T_{\text {eff }}=9011 \pm 85 \mathrm{~K}$ and luminosity $\log L / L_{\odot}=1.12 \pm 0.07$ dex.

Table 5

Observed Fluxes of the Spectral Energy Distribution of HD 1160 A

\begin{tabular}{lcc}
\hline \hline $\begin{array}{l}\text { Wavelength } \\
(\mu \mathrm{m})\end{array}$ & $\begin{array}{c}\text { Width } \\
(\mu \mathrm{m})\end{array}$ & $\begin{array}{c}\text { Flux } \\
\left(\mathrm{erg} / \mathrm{s} / \mathrm{cm}^{-2} \times 10^{-10}\right)\end{array}$ \\
\hline 0.274 & 0.030 & $111.000 \pm 2.740$ \\
0.236 & 0.030 & $117.000 \pm 6.150$ \\
0.197 & 0.030 & $130.000 \pm 8.250$ \\
0.157 & 0.030 & $67.000 \pm 4.230$ \\
0.422 & 0.145 & $403.361 \pm 3.715$ \\
0.535 & 0.167 & $317.359 \pm 2.923$ \\
0.358 & 0.062 & $224.376 \pm 2.067$ \\
0.450 & 0.091 & $423.056 \pm 3.896$ \\
0.556 & 0.086 & $289.911 \pm 2.670$ \\
0.357 & 0.056 & $198.323 \pm 1.827$ \\
0.487 & 0.129 & $367.277 \pm 3.383$ \\
0.626 & 0.134 & $227.942 \pm 2.099$ \\
0.765 & 0.137 & $155.036 \pm 1.428$ \\
0.907 & 0.140 & $112.999 \pm 1.041$ \\
1.273 & 0.152 & $58.606 \pm 0.540$ \\
1.671 & 0.237 & $26.964 \pm 0.248$ \\
2.171 & 0.255 & $13.514 \pm 0.124$ \\
3.353 & 0.663 & $4.753 \pm 0.232$ \\
4.603 & 1.042 & $1.745 \pm 0.031$ \\
11.561 & 5.507 & $0.112 \pm 0.010$ \\
22.800 & 4.101 & $0.014 \pm 0.002$ \\
\hline
\end{tabular}

Note. Fluxes from the literature used in the SED fitting of HD 1160 A, from Thompson et al. (1978), Cutri et al. (2003), Ammons et al. (2006), Pickles \& Depagne (2010), and Cutri et al. (2012). Column 3 corresponds to the red data points in Figure 11.

there is no need to posit a subsolar metallicity for the system. $^{46}$

\footnotetext{
$\overline{46}$ A substantially subsolar metallicity is unlikely for other reasons. Nearby young star clusters are nearly all within $[\mathrm{Fe} / \mathrm{H}] \approx 0.1$ dex of solar metallicity (see the continually updated catalog from Dias et al. 2002). The youngest nearby star-forming regions may be slightly sub-solar, $([\mathrm{Fe} / \mathrm{H}] \approx-0.1)$ but with very little dispersion (Santos et al. 2008); slightly older moving groups like $\mathrm{AB}$ Dor appear slightly metal-rich $[\mathrm{Fe} / \mathrm{H}] \approx 0.1$ (Biazzo et al. 2012). Thus, the small dispersion in metallicity among young groups makes $[\mathrm{Fe} / \mathrm{H}]=$ -0.3 unlikely.
} 
Table 6

Age Estimations of the HD 1160 System

\begin{tabular}{|c|c|}
\hline Evidence & Age Constraint \\
\hline $\begin{array}{l}\text { HD } 1160 \mathrm{~A} \text { is a main-sequence } \\
\text { early A star }\end{array}$ & $\lesssim 300 \mathrm{Myr}$ \\
\hline Darthmouth Models & $\begin{array}{l}\text { 13-150 Myr for HD } 1160 \mathrm{~A} ;>50 \mathrm{Myr} \\
\text { for HD } 1160 \mathrm{C}\end{array}$ \\
\hline Yonsei-Yale Models & 20-80 Myr for A; >75 Myr for C \\
\hline Baraffe Models & $>80$ Myr for $\mathrm{B} ;>45 \mathrm{Myr}$ for $\mathrm{C}$ \\
\hline MIST Models & 20-125 Myr for $\mathrm{A}$; and $>70 \mathrm{Myr}$ for $\mathrm{C}$ \\
\hline \multirow{2}{*}{$\begin{array}{l}\text { Bayesian analysis of Geneva } \\
\text { Models }\end{array}$} & 58 Myr (best-estimated age) \\
\hline & $\begin{array}{l}\lesssim 200 \mathrm{Myr}(90 \% \text { confidence }) \text { for } \\
\text { HD } 1160 \text { A }\end{array}$ \\
\hline Adopted Age HD 1160 A only & $20-125 \mathrm{Myr}$ \\
\hline $\begin{array}{l}\text { Adopted Age HD } 1160 \text { A, B } \\
\text { and C }\end{array}$ & $80-125 \mathrm{Myr}$ \\
\hline
\end{tabular}

Note. Ages of HD 1160 system as shown in Figure 13 and detailed in Section 4.

Given that HD 1160 A has an unknown rotational velocity $v \sin i$, we also consider the effect of rotation on the derived ages. Rapidly rotating stars such as Altair, Vega, and substellar companion host Kappa And A exhibit temperature changes of $\sim 1000-2000 \mathrm{~K}$ from the stellar pole to equator. This effect is due to gravity darkening, which is directly observed via optical/IR interferometry (Monnier et al. 2007, 2012; Jones et al. 2016) and modeled extensively by Espinosa Lara \& Rieutord (2011). Given this large temperature change across the star's surface, only a rapidly rotating star's disk-integrated apparent luminosity is observable, which varies with inclination. Jones et al. (2016) observed a 16\% difference in the true and apparent luminosity of Kappa And A, a difference that impacts the star's best-estimated age. HD 1160 A may be a rapidly rotating star, and thus only its apparent luminosity and apparent effective temperature are directly observable without interferometry. Therefore, we derive a Bayesian age estimation for HD 1160 A from the Brandt \& Huang (2015) server ${ }^{47}$ which incorporates these uncertainties. Brandt \& Huang (2015) 's method utilizes the Geneva evolutionary models (Georgy et al. 2013), the atmosphere models of Castelli \& Kurucz (2004), and orientation-dependent effective temperatures of Espinosa Lara \& Rieutord (2011).

\subsection{Results}

Our comparisons of HD 1160 to evolutionary models are shown in Figures 12 and 13. Evolutionary models suggest a young age for HD 1160 A: 13-150 Myr, 20-80 Myr, and 20-125 Myr for the Dartmouth, Yale-Yonsei, and MIST models, respectively, where we quote age ranges consistent with 1- $\sigma$ uncertainties in temperature and luminosity. The Bayesian techniques of Brandt \& Huang (2015) also point toward a young age. Bayesian methods are consistent with this estimate, with a "best-estimated" age (mean of the age $\times$ probability) of $\sim 58 \mathrm{Myr}$, and reject ages $\gtrsim 200 \mathrm{Myr}$ at $90 \%$ confidence.

Model comparisons to HD 1160's lower-mass components generally favor older ages. The Dartmouth, Yale-Yonsei, MIST, and Baraffe models favor ages of $>50 \mathrm{Myr}$,

\footnotetext{
47 www.bayesianstellarparameters.info
}

$>75 \mathrm{Myr},>70 \mathrm{Myr}$, and >45 Myr for HD $1160 \mathrm{C}$. The Baraffe models imply an age of $>80$ Myr for HD 1160 B. In most cases, the lower limit on the components' luminosity implies stellar objects on/near the main sequence, precluding us from assigning age upper limits.

Therefore, considering HD 1160 A alone, we derive an age range of $\approx 20-125 \mathrm{Myr}$ for the system. Considering HD 1160 ABC jointly implies a system age of $80-125$ Myr. That much of the model age ranges for HD 1160 A versus HD $1160 \mathrm{BC}$ do not overlap at the 1- $\sigma$ level (Figure 13) may suggest discrepancies between models and the true physical properties of young M-dwarfs (Feiden et al. 2011; Kraus et al. 2015).

\section{MASSES OF HD 1160 B AND C}

Given an age of 80-125 Myr from analyzing HD 1160 A, B and $\mathrm{C}$ jointly, and luminosities of $\log L / L_{\odot}=-2.76 \pm 0.05$ and $\log L / L_{\odot}=-2.16 \pm 0.04$, we derive mass ranges of 70-90 $M_{\mathrm{J}}$ and 175-200 $M_{\mathrm{J}}$ for HD 1160 B and C respectively, using the Baraffe et al. (2015) models. Considering instead the age range derived from HD $1160 \mathrm{~A}$ alone, we estimate masses of 35-90 $M_{\mathrm{J}}$ for B and 110-200 $M_{\mathrm{J}}$ for HD $1160 \mathrm{C}$.

Mass estimates derived from our atmospheric modeling are broadly consistent with those derived from luminosity evolution. The best-fit radius and surface gravity for HD $1160 \mathrm{~B}$ in turn imply a mass of $\sim 29_{-20.8}^{+63.8} M_{\mathrm{J}}$. Surface gravity is the key factor, as models with $\log g=4.0$ imply a planet-mass companion $\left(8-9 M_{\mathrm{J}}\right)$, those with $\log g=4.5$ imply a $\sim 30 M_{\mathrm{J}}$ brown dwarf, and those with $\log g=5.0$ imply an object slightly above the hydrogen burning limit of 70-80 $M_{\mathrm{J}}$ (Dieterich et al. 2014). ${ }^{48}$

Thus, the interpretation of HD $1160 \mathrm{~B}$ changes depending on which system component(s) we use to estimate an age. Using only HD 1160 A to calibrate the system's age, HD 1160 B's allowable mass range mostly covers the substellar regime. But ages derived from both HD $1160 \mathrm{~A}, \mathrm{~B}$ and $\mathrm{C}$ preclude the youngest ages (20-80 Myr), and suggest that HD 1160 B straddles the hydrogen burning limit.

Our mass ranges for HD 1160 B agree with those of M16, although our mass upper limit is smaller due to our somewhat lower upper age limit of $\sim 125 \mathrm{Myr}$ for the HD 1160 system. However, our mass ranges for HD $1160 \mathrm{~B}$ are wider than the mass range of 24-45 $M_{\mathrm{J}}$ from N12 using $J$ band photometry, and generally skew toward larger values.

\section{CONCLUSIONS}

We present new and precise SCExAO NIR photometry, reprocessed thermal infrared photometry, and $Y$ band GPI integral field spectroscopy of the HD 1160 system, which hosts two low-mass companions discovered by N12. Combining our data with recently published NIR spectroscopy from SPHERE, we constrain the infrared colors of HD $1160 \mathrm{BC}$ and constrain the atmospheric properties of HD $1160 \mathrm{~B}$. After revisiting the age of the HD 1160 system using multiple diagnostics, we determine the likely intrinsic properties of HD $1160 \mathrm{~B}$ and C.

\footnotetext{
${ }^{48}$ As a point of comparison, the surface gravity predicted for HD 1160 B by the Baraffe models for a $30 \mathrm{Myr}$ old, $35 M_{\mathrm{J}}$ object is $\log g \sim 4.4$ and $\mathrm{fpr}$ a $125 \mathrm{Myr}$ old, $90 M_{\mathrm{J}}$ object is $\log g \sim 4.9$. While a subset of these surface gravities are consistent with results from our atmospheric modeling, the predicted temperatures are significantly lower $(2600-2850 \mathrm{~K})$, discrepancies that may point to additional challenges in predicting the evolution of young substellar objects and the lowest-mass stars (see also Kraus et al. 2015).
} 

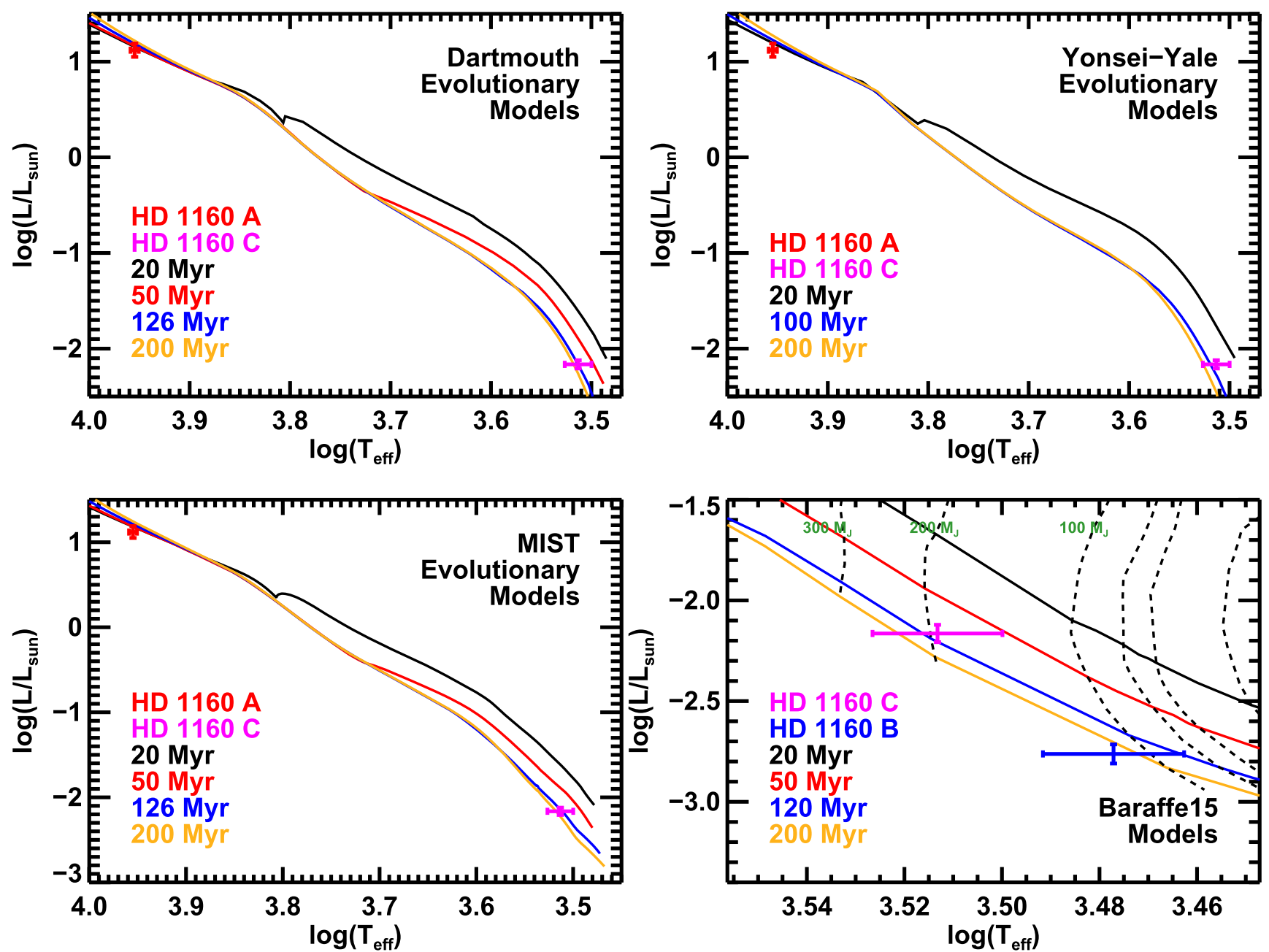

Figure 12. A comparison of the effective temperature and luminosities of HD 1160 A (red point, derived from SED fitting in Figure 11), and HD 1160 C (light blue point, see Section 3.5) to stellar models. The Dartmouth, Yonsei-Yale, and MIST evolutionary models do not extend into the substellar $\left(<0.1 M_{\odot}\right)$ regime, precluding a reliable age estimate for HD $1160 \mathrm{~B}$, while the Baraffe15 models do not extend to the mass of HD $1160 \mathrm{~A}$.

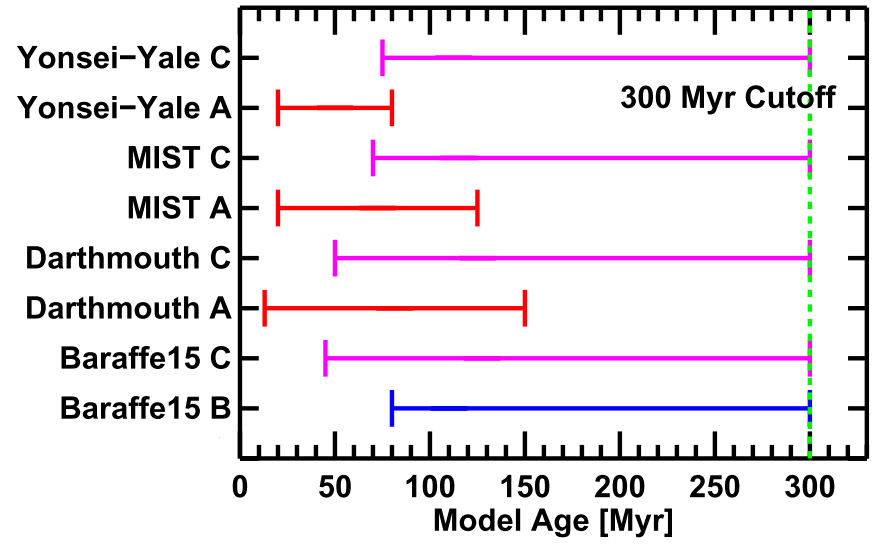

Figure 13. Ages derived from the Baraffe15, DSEP, MIST, and Yonsei-Yale isochrones (see Figure 12) for HD $1160 \mathrm{~A}, \mathrm{~B}$, and C. The model ages are derived from the observed $T_{\text {eff }}$ and $\log L / L_{\odot}$ for each star. The bright green dotted line denotes the 300 Myr cut-off age for the system, given that HD 1160 A is a main-sequence star (Siess et al. 2000).

Our study yields the following results:

1. Our SCEXAO photometry refines estimates for HD 1160 B's brightness in major infrared passbands. Our photometry in general agrees with the SPHERE $1.0-1.6 \mu \mathrm{m}$ spectrum of M16, although it is systematically fainter by $15 \%-25 \%$ for HD 1160 B. Like M16, our SCExAO photometry for HD $1160 \mathrm{~B}$ is in strong disagreement with the $J$ and $H$ photometry of Nielsen et al. (2012) by 1.14 and 0.44 mag respectively; our re-derived $M^{\prime}$ photometry likewise shows differences.

2. These differences, in turn, lead to a reinterpretation of HD 1160 B and C's infrared colors. Specifically, HD 1160 $\mathrm{BC}$ are not discrepant or redder than the field sequence but completely consistent with the colors expected for field dwarfs with early-to-mid-M spectral types.

3. We revise the spectral type of HD $1160 \mathrm{~B}$ to M5.5 $5_{-0.5}^{+1.0}$, earlier than proposed by N12 based purely on NIR colors but generally consistent with the results from M16 based on longer-wavelength spectra.

4. Our atmospheric modeling finds a best-fit temperature for HD $1160 \mathrm{~B}$ of $3000-3100 \mathrm{~K}$ and a surface gravity of $\log g=4-4.5$, although we cannot rule out slightly cooler temperatures or slightly higher surface gravities over some portion of parameter space.

5. In contrast with the results from M16, there is no evidence from atmospheric modeling for HD $1160 \mathrm{~B}$ having a subsolar metallicity, as the best-fitting (solar 
metallicity) models are consistent with the data, having a reduced $\chi^{2} \sim 1$.

6. We find different answers for the system's age depending on which components are analyzed. Considering HD $1160 \mathrm{~A}, \mathrm{~B}$ and $\mathrm{C}$ jointly, we estimate an age of 80-125 Myr, implying masses of $70-90 M_{\mathrm{J}}$ for $\mathrm{HD}$ $1160 \mathrm{~B}$, making it an object straddling the hydrogen burning limit. Considering HD 1160 A alone, we estimate an age of $20-125 \mathrm{Myr}$, a wider range than that listed in $\mathrm{N} 12$ and younger than that estimated in M16. Given this age, we derive a mass of 35-90 $M_{\mathrm{J}}$ for HD $1160 \mathrm{~B}$. Estimated masses for HD $1160 \mathrm{~B}$ from atmospheric modeling are consistent with these ranges but allow for slightly lower masses. Our analysis is then consistent with HD 1160 B being a relatively intermediate-to-highmass brown dwarf and HD $1160 \mathrm{C}$ being a low-mass star.

HD 1160 is an important triple system, with one high-mass star, one very low-mass star, and one brown dwarf/extremely low-mass star. As such, it presents an excellent laboratory for studying (sub)stellar evolution and testing our understanding of luminosity evolution. Our results may hint at challenges ahead, as age estimates derived from HD $1160 \mathrm{~A}$, although formally consistent with those from HD $1160 \mathrm{BC}$, tend to be younger than those derived from the system's lower-mass companions. This trend is an inversion of that seen for ages derived for the youngest clusters/moving groups: e.g., ages derived for Upper Scorpius intermediate-mass stars are older than those derived for its lower-mass stars (e.g., Preibisch et al. 2002; Pecaut et al. 2012). Interferometric measurements of HD 1160 A's angular diameter, combined with a Gaia parallax more precise than Hipparcos, would clarify the primary's true luminosity and temperature, allowing us to place more firm constraints on its age based on its HR diagram position.

HD $1160 \mathrm{~B}$ is one of many young substellar objects that may provide key insights into young substellar object formation and atmospheres. Its mass ratio $(\sim 0.015-0.040$ or $0.027-0.040)$ perhaps indicates formation by protostellar disk fragmentation/ disk instability (e.g., Boss 2011), or is consistent with the lowest-mass objects formed like binary stars (Reggiani \& Meyer 2011). Indeed, HD 1160 B's mass range and the semimajor axis is compatible with a disk-instability scenario of formation, provided the disk is $\sim 50 \%$ the star's mass (Figure C.1 in Bonnefoy et al. 2014).

As shown by works like Currie et al. (2013, 2014a), and most recently (and exhaustively) by Faherty et al. (2016), low-gravity L- and T-type brown dwarfs and directly imaged planets across a wide range of spectral types and temperatures appear to depart from the field dwarf sequence. HD $1160 \mathrm{~B}$, an earlier spectral type object, does not yet show differences. Identifying where/ why young and low-gravity objects begin to depart from the field provides some insight into, and an empirical constraint, on the evolution of substellar atmospheres.

While the masses inferred for HD 1160 B from atmospheric modeling appear consistent with those implied by luminosity evolution models, our results hint that follow-up spectroscopy could better constrain the object's surface gravity and (by inference) mass. The implied mass of HD $1160 \mathrm{~B}$ varies by a factor of ten from the lowest to highest surface gravity from our suite of acceptably fitting models. As described in Allers \& Liu (2013), higher-resolution spectroscopy focused on line transitions such as Na I and K I could better clarify how HD 1160 B's surface gravity compares to that of other brown dwarfs with a range of ages. Facilities such as Keck/OSIRIS and VLT/ SINFONI could potentially provide these data.

We thank Anna-Lise Marie for detailed, helpful discussions about and access to SPHERE HD $1160 \mathrm{~B}$ spectra. We thank Eric Mamajek for discussions about the metallicity of nearby, young stars. We thank Davy Kirkpatrick for providing NIR spectra of M-dwarf spectral standards. We thank Federico Spada for discussions and tests of the Yonsei-Yale models. EVG would like to acknowledge the gracious support of his Lowell Predoctoral Fellowship by the BF foundation and the excellent support of Fisk-Vanderbilt Bridge Program. This research has made use of the SIMBAD database, operated at CDS, Strasbourg, France. This research has made use of the Keck Observatory Archive (KOA), which is operated by the W. M. Keck Observatory and the NASA Exoplanet Science Institute (NExScI), under contract with the National Aeronautics and Space Administration. The authors acknowledge support from the JSPS (Grant-in-Aid for Research \#23103002, \#23340051, and \#26220704). This work was partially supported by the Grant-inAid for JSPS fellows (Grant Number 25-8826). M.J. acknowledges support of the U.S. National Science Foundation, under Award No. 1009203. This work performed under the auspices of the U.S. Department of Energy by Lawrence Livermore National Laboratory under Contract DE-AC52-07NA27344 with document release number LLNL-JRNL-701012-DRAFT. We wish to acknowledge the pivotal cultural role and reverence that the summit of Maunakea has always had within the indigenous Hawaiian community. We are most fortunate to have the privilege to conduct scientific observations from this mountain.

\section{REFERENCES}

Allard, F., Homeier, D., Freytag, B., \& Sharp, C. M. 2012, RSPTA, 370, 2765 Allers, K. N., \& Liu, M. C. 2013, ApJ, 772, 79

Ammons, S. M., Robinson, S. E., Strader, J., et al. 2006, ApJ, 638, 1004

Baraffe, I., Chabrier, G., Barman, T. S., Allard, F., \& Hauschildt, P. H. 2003, A\&A, 402, 701

Baraffe, I., Homeier, D., Allard, F., \& Chabrier, G. 2015, A\&A, 577, A42 Beuzit, J.-L., Feldt, M., Dohlen, K., et al. 2008, Proc. SPIE, 7014, 701418

Biazzo, K., D’Orazi, V., Desidera, S., et al. 2012, MNRAS, 427, 2905 Biller, B. A., Liu, M. C., Wahhaj, Z., et al. 2010, ApJL, 720, L82

Boccaletti, A., Lagrange, A.-M., Bonnefoy, M., Galicher, R., \& Chauvin, G. 2013, A\&A, 551, L14

Bonnefoy, M., Currie, T., Marleau, G.-D., et al. 2014, A\&A, 562, A111

Boss, A. P. 2011, ApJ, 731, 74

Brandt, T. D., \& Huang, C. X. 2015, ApJ, 807, 58

Carson, J., Thalmann, C., Janson, M., et al. 2013, ApJL, 763, L32

Castelli, F., \& Kurucz, R. L. 2004, arXiv:astro-ph/0405087

Chauvin, G., Lagrange, A.-M., Zuckerman, B., et al. 2005, A\&A, 438, L29

Choi, J., Dotter, A., Conroy, C., et al. 2016, ApJ, 823, 102

Currie, T., Burrows, A., Itoh, Y., et al. 2011, ApJ, 729, 128

Currie, T., Burrows, A., Madhusudhan, N., et al. 2013, ApJ, 776, 15

Currie, T., Cloutier, R., Brittain, S., et al. 2015, ApJL, 814, L27

Currie, T., Daemgen, S., Debes, J., et al. 2014a, ApJL, 780, L30

Currie, T., Muto, T., Kudo, T., et al. 2014b, ApJL, 796, L30

Cutri, R. M., Skrutskie, M. F., van Dyk, S., et al. 2003, yCat, 2246, 0

Cutri, R. M., Wright, E. L., Conrow, T., et al. 2012, yCat, 2311, 0

Dawson, P., Scholz, A., Ray, T. P., et al. 2014, MNRAS, 442, 1586

Dias, W. S., Alessi, B. S., Moitinho, A., \& Lépine, J. R. D. 2002, A\&A, 389, 871

Dieterich, S. B., Henry, T. J., Jao, W.-C., et al. 2014, AJ, 147, 94

Dotter, A. 2016, ApJS, 222, 8

Dotter, A., Chaboyer, B., Jevremović, D., et al. 2008, ApJS, 178, 89

Dupuy, T. J., Forbrich, J., Rizzuto, A., et al. 2016, ApJ, 827, 23

Dupuy, T. J., \& Liu, M. C. 2012, ApJS, 201, 19

Dupuy, T. J., Liu, M. C., Bowler, B. P., et al. 2010, ApJ, 721, 1725

Dupuy, T. J., Liu, M. C., \& Ireland, M. J. 2009, ApJ, 692, 729

Dupuy, T. J., Liu, M. C., Leggett, S. K., et al. 2015, ApJ, 805, 56

Elias, J. H., Frogel, J. A., Matthews, K., \& Neugebauer, G. 1982, AJ, 87, 1029 
Espinosa Lara, F., \& Rieutord, M. 2011, A\&A, 533, A43

Faherty, J. K., Riedel, A. R., Cruz, K. L., et al. 2016, ApJS, 225, 10

Feiden, G. A., Chaboyer, B., \& Dotter, A. 2011, ApJL, 740, L25

Galicher, R., Marois, C., Macintosh, B., Barman, T., \& Konopacky, Q. 2011, ApJL, 739, L41

Georgy, C., Ekström, S., Granada, A., et al. 2013, A\&A, 553, A24

Hauschildt, P. H., Allard, F., \& Baron, E. 1999, ApJ, 512, 377

Hewett, P. C., Warren, S. J., Leggett, S. K., \& Hodgkin, S. T. 2006, MNRAS, 367,454

Ireland, M. J., Kraus, A., Martinache, F., Law, N., \& Hillenbrand, L. A. 2011, ApJ, 726, 113

Jones, J., White, R. J., Quinn, S., et al. 2016, ApJL, 822, L3

Jovanovic, N., Martinache, F., Guyon, O., et al. 2015, PASP, 127, 890

Kirkpatrick, J. D., Looper, D. L., Burgasser, A. J., et al. 2010, ApJS, 190, 100

Konopacky, Q. M., Thomas, S. J., Macintosh, B. A., et al. 2014, Proc. SPIE, 9147, 84

Kratter, K. M., Murray-Clay, R. A., \& Youdin, A. N. 2010, ApJ, 710, 1375

Kraus, A. L., Cody, A. M., Covey, K. R., et al. 2015, ApJ, 807, 3

Kraus, A. L., \& Ireland, M. J. 2012, ApJ, 745, 5

Kuzuhara, M., Tamura, M., Kudo, T., et al. 2013, ApJ, 774, 11

Lagrange, A.-M., Bonnefoy, M., Chauvin, G., et al. 2010, Sci, 329, 57

Larkin, J. E., Chilcote, J. K., Aliado, T., et al. 2014, Proc. SPIE, 9147, 1

Liu, M. C., Dupuy, T. J., Bowler, B. P., Leggett, S. K., \& Best, W. M. J. 2012, ApJ, 758, 57

Liu, M. C., Wahhaj, Z., Biller, B. A., et al. 2010, Proc. SPIE, 7736, 1

Lozi, J., Guyon, O., Jovanovic, N., et al. 2016, Proc. SPIE, 9909, 9909

Macintosh, B., Graham, J., Palmer, D., et al. 2006, Proc. SPIE, 6272, 0

Macintosh, B., Graham, J. R., Barman, T., et al. 2015, Sci, 350, 64

Macintosh, B. A., Anthony, A., Atwood, J., et al. 2014, Proc. SPIE, 9148, 0

Maire, A.-L., Bonnefoy, M., Ginski, C., et al. 2016, A\&A, 587, A56

Maire, J., Perrin, M. D., Doyon, R., et al. 2010, Proc. SPIE, 7735, 31

Maire, J., Perrin, M. D., Doyon, R., et al. 2012, Proc. SPIE, 8451, 3

Marois, C., Lafrenière, D., Doyon, R., Macintosh, B., \& Nadeau, D. 2006, ApJ, 641, 556

Marois, C., Macintosh, B., Barman, T., et al. 2008, Sci, 322, 1348

Marois, C., Macintosh, B., \& Véran, J.-P. 2010, Proc. SPIE, 7736, 77361J
Monnier, J. D., Che, X., Zhao, M., et al. 2012, ApJL, 761, L3

Monnier, J. D., Zhao, M., Pedretti, E., et al. 2007, Sci, 317, 342

Nielsen, E. L., Liu, M. C., Wahhaj, Z., et al. 2012, ApJ, 750, 53

Paxton, B., Bildsten, L., Dotter, A., et al. 2011, ApJS, 192, 3

Paxton, B., Cantiello, M., Arras, P., et al. 2013, ApJS, 208, 4

Pecaut, M. J., \& Mamajek, E. E. 2013, ApJS, 208, 9

Pecaut, M. J., Mamajek, E. E., \& Bubar, E. J. 2012, ApJ, 746, 154

Perrin, M. D., Maire, J., Ingraham, P., et al. 2014, Proc. SPIE, 9147, 3

Pickles, A., \& Depagne, É. 2010, PASP, 122, 1437

Pickles, A. J. 1998, PASP, 110, 863

Preibisch, T., Brown, A. G. A., Bridges, T., Guenther, E., \& Zinnecker, H. 2002, AJ, 124, 404

Press, W. H., Teukolsky, S. A., Vetterling, W. T., \& Flannery, B. P. 1992, Numerical Recipes in FORTRAN. The Art of Scientific Computing (Cambridge: Cambridge Univ. Press)

Quanz, S. P., Amara, A., Meyer, M. R., et al. 2013, ApJL, 766, L1

Rameau, J., Chauvin, G., Lagrange, A.-M., et al. 2013, ApJL, 779, L26

Rayner, J. T., Toomey, D. W., Onaka, P. M., et al. 2003, PASP, 115, 362

Reggiani, M. M., \& Meyer, M. R. 2011, ApJ, 738, 60

Santos, N. C., Melo, C., James, D. J., et al. 2008, A\&A, 480, 889

Siess, L., Dufour, E., \& Forestini, M. 2000, A\&A, 358, 593

Soderblom, D. R., Hillenbrand, L. A., Jeffries, R. D., Mamajek, E. E., \& Naylor, T. 2014, in Protostars and Planets VI, ed. H. Beuther et al. (Tucson, AZ: Univ. of Arizona Press), 219

Soummer, R., Pueyo, L., \& Larkin, J. 2012, ApJL, 755, L28

Spada, F., Demarque, P., Kim, Y.-C., \& Sills, A. 2013, ApJ, 776, 87

Tamura, M. 2009, in AIP Conf. Ser. 1158, Subaru Strategic Exploration of Exoplanets and Disks with HiCIAO/AO188 (SEEDS), ed. T. Usuda, M. Tamura, \& M. Ishii (Melville, NY: AIP), 11

Thompson, G. I., Nandy, K., Jamar, C., et al. 1978, Catalogue of Stellar Ultraviolet Fluxes. A Compilation of Absolute Stellar Fluxes Measured by the Sky Survey Telescope (S2/68) Aboard the ESRO Satellite TD-1 (London: Sci. Res. Council)

van Leeuwen, F. 2007, A\&A, 474, 653

Wang, J. J., Rajan, A., Graham, J. R., et al. 2014, Proc. SPIE, 9147, 55

Yi, S. K., Kim, Y.-C., \& Demarque, P. 2003, ApJS, 144, 259 